\title{
Systematic assessment of long-read RNA-seq methods for transcript identification and quantification
}

\section{Francisco Pardo-Palacios}

Polytechnical University of Valencia https://orcid.org/0000-0002-3067-0166

\section{Fairlie Reese}

University of California, Irvine

\section{Silvia Carbonell-Sala}

Centre for Genomic Regulation (CRG), The Barcelona Institute of Science and Technology

\section{Mark Diekhans}

University of California, Santa Cruz https://orcid.org/0000-0002-0430-0989

\section{Cindy Liang}

University of California, Santa Cruz

\section{Dingjie Wang}

The Ohio State University

\section{Brian Williams}

California Institute of Technology https://orcid.org/0000-0003-3253-611X

\section{Matthew Adams}

University of California, Santa Cruz https://orcid.org/0000-0001-6793-6557

\section{Amit Behera}

University of California, Santa Cruz

\section{Julien Lagarde}

Centre for Genomic Regulation (CRG), The Barcelona Institute of Science and Technology

Haoran Li

The Ohio State University

\section{Andrey Pribelski}

St. Petersburg State University

\section{Gabriela Balderrama-Gutierrez}

University of California, Irvine

Muhammed Hasan Çelik

University of California, Irvine

\section{Maite De María}

University of Florida

\section{Nancy Denslow}


University of Florida https://orcid.org/0000-0002-3946-3112

\section{Natàlia Garcia-Reyero}

US Army Engineer Research \& Development Center

\section{Stefan Goetz}

Biobam Bioinformatics SL

\section{Margaret Hunter}

U.S. Geological Survey, Wetland, and Aquatic Research Center

\section{Jane Loveland}

European Molecular Biology Laboratory, European Bioinformatics Institute, EMBL-EBI, Wellcome Genome Campus

\section{Carlos Menor}

Biobam Bioinformatics SL

\section{David Moraga}

University of Florida

\section{Jonathan Mudge}

European Molecular Biology Laboratory, European Bioinformatics Institute, Wellcome Genome Campus

\section{Hazuki Takahashi}

$$
\text { RIKEN }
$$

\section{Alison Tang}

University of California, Santa Cruz

\section{Ingrid Youngworth}

Stanford University

\section{Piero Carninci}

RIKEN, Center for Integrative Medical Sciences https://orcid.org/0000-0001-7202-7243

\section{Roderic Guigó}

Pompeu Fabra University

\section{Hagen Tilgner}

Weill Cornell Medicine

\section{Barbara Wold}

California Institute of Technology https://orcid.org/0000-0003-3235-8130

\section{Christopher Vollmers}

University of California, Santa Cruz

\section{Gloria Sheynkman}

University of Virginia

\section{Adam Frankish}

European Bioinformatics Institute

\section{Kin Fai Au}

Department of Biomedical Informatics, The Ohio State University https://orcid.org/0000-0002-9222- 


\section{Ana Conesa}

Spanish National Research Council (CSIC)

\section{Ali Mortazavi}

University of California, Irvine https://orcid.org/0000-0002-4259-6362

\section{Angela N. Brooks ( $\square$ anbrooks@ucsc.edu )}

University of California, Santa Cruz https://orcid.org/0000-0002-7898-3073

\section{Analysis}

Keywords: long-read RNA, sequence methods, LRGASP, transcriptome analyses

Posted Date: August 3rd, 2021

DOI: https://doi.org/10.21203/rs.3.rs-777702/v1

License: (c) (i) This work is licensed under a Creative Commons Attribution 4.0 International License.

Read Full License 


\section{Systematic assessment of long-read RNA-seq methods for transcript 2 identification and quantification}

3 Francisco J. Pardo-Palacios ${ }^{1,29}$, Fairlie Reese ${ }^{2,3,29}$, Sílvia Carbonell-Sala ${ }^{4,29}$, Mark Diekhans ${ }^{5,29}$, 4 Cindy E. Liang ${ }^{6,29}$, Dingjie Wang ${ }^{7,29}$, Brian Williams ${ }^{8,29}$, Matthew S. Adams ${ }^{6}$, Amit K. Behera ${ }^{9}$, 5 Julien Lagarde $^{4}$, Haoran Li ${ }^{7}$, Andrey D. Prjibelski ${ }^{10}$, Gabriela Balderrama-Gutierrez ${ }^{2,3}$, 6 Muhammed Hasan Çelik ${ }^{2,3}$, Maite De María ${ }^{11,12}$, Nancy Denslow ${ }^{13}$, Natàlia Garcia-Reyero ${ }^{14}$, 7 Stefan Goetz ${ }^{15}$, Margaret E. Hunter ${ }^{16}$, Jane E. Loveland ${ }^{17}$, Carlos Menor ${ }^{15}$, David Moraga ${ }^{18}$, 8 Jonathan M. Mudge ${ }^{17}$, Hazuki Takahashi ${ }^{19}$, Alison D. Tang ${ }^{9}$, Ingrid Ashley. Youngworth ${ }^{20}$, Piero 9 Carninci $^{19,21}$, Roderic Guigó ${ }^{4,22}$, Hagen U. Tilgner ${ }^{23}$, Barbara J. Wold ${ }^{8}$, Christopher Vollmers ${ }^{9,30}$, 10 Gloria M. Sheynkman ${ }^{24,25,26,30}$, Adam Frankish ${ }^{17,30}$, Kin Fai $\mathrm{Au}^{7,30}$, Ana Conesa ${ }^{27,28,30^{*}}$, Ali 11 Mortazavi $^{2,3,30^{*}}$, Angela N. Brooks ${ }^{5,9,30^{*}}$

${ }^{1}$ Department of Applied Statistics and Operational Research and Quality, Polytechnical University of Valencia, Valencia, Spain, ${ }^{2}$ Developmental and Cell Biology, ${ }^{3}$ Center for Complex Biological Systems, University of California, Irvine, Irvine, USA, ${ }^{4}$ Centre for Genomic Regulation (CRG), The Barcelona Institute of Science and Technology, Dr. Aiguader 88, Barcelona 08003, Catalonia, Spain, ${ }^{5}$ UC Santa Cruz Genomics Institute, ${ }^{6}$ Molecular Cell and Developmental Biology, University of California, Santa Cruz, Santa Cruz, USA, ${ }^{7}$ Department of Biomedical Informatics, The Ohio State University, Columbus, USA, ${ }^{8}$ Division of Biology and Biological Engineering, California Institute of Technology, Pasadena, USA, ${ }^{9}$ Department of Biomolecular Engineering, University of California, Santa Cruz, Santa Cruz, USA, ${ }^{10} \mathrm{Center}$ for Bioinformatics and Algorithmic Biotechnology, Institute of Translational Biomedicine, St. Petersburg State University, St. Petersburg, Russia, ${ }^{11}$ Department of Physiological Sciences, College of Veterinary Medicine, ${ }^{12}$ Center for Environmental and Human Toxicology, ${ }^{13}$ Department of Physiological Sciences, Center for Environmental and Human Toxicology, University of Florida, Gainesville, USA, ${ }^{14}$ Environmental Laboratory, US Army Engineer Research \& Development Center, Vicksburg, USA, ${ }^{15}$ Biobam Bioinformatics SL, Valencia, Spain, ${ }^{16}$ U.S. Geological Survey, Wetland, and Aquatic Research Center, Gainesville, USA, ${ }^{17}$ European Molecular Biology Laboratory, European Bioinformatics Institute, Wellcome Genome Campus, Hinxton, Cambridge CB10 1SD, UK, ${ }^{18}$ Interdisciplinary Center for Biotechnology Research, University of Florida, Gainesville, USA, ${ }^{19}$ Center for Integrative Medical Sciences, Laboratory for Transcriptome Technology, RIKEN, Yokohama, Japan, ${ }^{20}$ Department of Genetics, Stanford University, Palo Alto, USA, ${ }^{21}$ Human Technopole, Milano, Italy, ${ }^{22}$ Universitat Pompeu Fabra (UPF), Barcelona, Catalonia, Spain, ${ }^{23}$ Brain and Mind Research Institute and Center for Neurogenetics, Weill Cornell Medicine, New York City, USA, ${ }^{24}$ Department of Molecular Physiology and Biological Physics, ${ }^{25}$ Center for Public Health Genomics, ${ }^{26}$ UVA Cancer Center, University of Virginia, Charlottesville, USA, ${ }^{27}$ Institute for Integrative Systems Biology, Spanish National Research Council (CSIC), Paterna, Spain, Gainesville, USA, ${ }^{29}$ These authors contributed equally, ${ }^{30}$ These authors jointly supervised the work, ${ }^{*}$ correspondence: 


\section{Abstract}

38 With increased usage of long-read sequencing technologies to perform transcriptome analyses, there becomes a greater need to evaluate different methodologies including library preparation, sequencing platform, and computational analysis tools. Here, we report the study design of a community effort called the Long-read RNA-Seq Genome Annotation Assessment Project (LRGASP) Consortium, whose goals are characterizing the strengths and remaining challenges in using long-read approaches to identify and quantify the transcriptomes of both model and non-model organisms. The LRGASP organizers have generated cDNA and direct RNA datasets in human, mouse, and manatee samples using different protocols followed by sequencing on Illumina, Pacific Biosciences, and Oxford Nanopore Technologies platforms. Participants will use the provided data to submit predictions for three challenges: transcript isoform detection with a high-quality genome, transcript isoform quantification, and de novo transcript isoform identification. Evaluators from different institutions will determine which pipelines have the highest accuracy for a variety of metrics using benchmarks that include spike-in synthetic transcripts, simulated data, and a set of undisclosed, manually curated transcripts by GENCODE. We also describe plans for experimental validation of predictions that are platformspecific and computational tool-specific. We believe that a community effort to evaluate longread RNA-seq methods will help move the field toward a better consensus on the best approaches to use for transcriptome analyses.

\section{Introduction}

58 There is a growing trend of using long-read RNA-seq (IrRNA-seq) data for transcript identification and quantification, primarily with Oxford Nanopore Technologies (ONT) and Pacific Biosciences (PacBio) platforms ${ }^{1-4}$. Consequently, there is a need to evaluate these approaches for transcriptome analysis to compare the impact of different sequencing platforms, multiple sequencing library preparation methods, and computational analysis methods (Reviewed in ${ }^{5-8}$ ).

64 A previous effort by the RNA-Seq Genome Annotation Assessment Project (RGASP)

65 Consortium ${ }^{9,10}$ involved evaluating short-read Illumina RNA-seq for transcript identification and

66 revealed limitations in recalling full-length transcript products due to the complexity of eukaryotic

67 transcriptomes. Although IrRNA-seq should improve transcript reconstruction, at a fixed cost, 
the reduced sequencing depth and higher error rates of long-read sequencing approaches may offset the improvements.

To evaluate long-read approaches for transcriptome analysis, we formed the Long-read RNA-

72 Seq Genome Annotation Assessment Project (LRGASP) Consortium modeled after the

73 previous $\mathrm{GASP}^{11}, \mathrm{EGASP}^{12}$, and $\mathrm{RGASP}^{9,10}$ efforts. For this project, we aim for an open

74 community effort in order to be as transparent and inclusive as possible in evaluating

75 technologies and computational methods (Fig 1).

The LRGASP Consortium will evaluate three fundamental aspects of transcriptome analysis.

78 First, we will assess the reconstruction of full-length transcripts expressed in a given sample

79 from a well-curated eukaryotic genome such as human and mouse. Second, we will evaluate

80 the quantification of the abundance of each transcript. Finally, we will assess de novo

81 reconstruction of full-length transcripts from samples without a high-quality genome, which

82 would be beneficial for annotating genes in non-model organisms. These evaluations became

83 the basis of the three challenges that comprise the LRGASP effort (Box 1).

\section{Challenge 1: Transcript isoform detection with a high-quality genome}

Goal: Identify which sequencing platform, library prep, and computational tool(s) combination gives the highest sensitivity and precision for transcript detection.

\section{Challenge 2: Transcript isoform quantification}

Goal: Identify which sequencing platform, library prep, and computational tool(s) combination gives the most accurate expression estimates.

\section{Challenge 3: De novo transcript isoform identification}

Goal: Identify which sequencing platform, library prep, and computational tool(s) combination gives the highest sensitivity and precision for transcript detection without a high-quality annotated genome.

Box 1: Overview of the LRGASP Challenges

87 The LRGASP Challenges will use data produced by the LRGASP Consortium Organizers (Fig

88 1b, Table 1, Supplementary Table 1). The samples for Challenges 1 and 2 consist of human 
and mouse ENCODE biosamples with extensive chromatin-level functional data generated separately by the ENCODE Consortium. These include the human WTC-11 iPSC cell line and a mouse 129/Casteneus ES cell line for Challenge 1 and a mix of $\mathrm{H} 1$ and Definitive Endoderm derived from $\mathrm{H} 1$ (H1-DE) for Challenge 2. In addition, individual $\mathrm{H} 1$ and $\mathrm{H} 1-\mathrm{DE}$ samples are

93 being sequenced on all platforms; however, those reads will not be released until after the end

94 of the challenge. All samples were grown as biological triplicates with the RNA extracted at one

95 site, spiked with 5'-capped Spike-In RNA Variants (Lexogen SIRV-Set 4), and distributed to all 96 production groups. After sequencing, reads for human and mouse samples were deposited at 97 the ENCODE Data Coordination Center (DCC) for community access, including but not limited 98 to the challenges. A single replicate of manatee whole blood transcriptome was generated for 99 Challenge 3. For each sample, we performed different CDNA preparation methods, including an 100 early-access ONT cDNA kit (PCS110), ENCODE PacBio CDNA, R2C2 ${ }^{13}$ for increased sequence 101 accuracy of ONT data, and CapTrap to enrich for 5'-capped RNAs. CapTrap is derived from the 102 CAGE technique ${ }^{14}$ and was adapated for IrRNA-seq (manuscript in preparation). We also 103 performed direct RNA sequencing (dRNA) with ONT.

\begin{tabular}{|c|c|c|c|c|c|c|c|c|}
\hline Sample & $\begin{array}{l}\text { \# of } \\
\text { Reps }\end{array}$ & $\begin{array}{l}\text { PacBio } \\
\text { cDNA }\end{array}$ & $\begin{array}{l}\text { ONT } \\
\text { cDNA }\end{array}$ & $\begin{array}{l}\text { ONT } \\
\text { direct } \\
\text { RNA }\end{array}$ & $\mathrm{R} 2 \mathrm{C} 2$ & $\begin{array}{l}\text { CapTrap } \\
\text { PacBio }\end{array}$ & $\begin{array}{l}\text { CapTrap } \\
\text { ONT }\end{array}$ & $\begin{array}{l}\text { Illumina } \\
\text { cDNA }\end{array}$ \\
\hline $\begin{array}{l}\text { Mouse } 129 / \text { Cast } \\
\text { ES cell line }\end{array}$ & 3 & Yes & Yes & Yes & Yes & Yes & Yes & Yes \\
\hline Human WTC-11 & 3 & Yes & Yes & Yes & Yes & Yes & Yes & Yes \\
\hline $\begin{array}{l}\text { Human H1 } \\
\text { ES/Definitive } \\
\text { Endoderm cell } \\
\text { line mix }\end{array}$ & 3 & Yes & Yes & Yes & Yes & Yes & Yes & Yes \\
\hline $\begin{array}{l}\text { Human H1 ES } \\
\text { cell line }\end{array}$ & 3 & Yes & Yes & Yes & Yes & Yes & Yes & Yes \\
\hline $\begin{array}{l}\text { Human H1 } \\
\text { Definitive } \\
\text { Endoderm cell } \\
\text { line }\end{array}$ & 3 & Yes & Yes & Yes & Yes & Yes & Yes & Yes \\
\hline $\begin{array}{l}\text { Trichechus } \\
\text { manatus } \\
\text { peripheral } \\
\text { blood } \\
\text { mononuclear } \\
\text { cells }\end{array}$ & 1 & Yes & Yes & No & No & No & No & Yes \\
\hline
\end{tabular}


Table 1: Overview of LRGASP sequencing data. The $\mathrm{H} 1$ and $\mathrm{H} 1$ Definitive Endoderm samples are sequenced but are not available to participants until the close of challenges.

Participants may provide multiple submissions for each challenge (detailed in Challenge submissions and timeline) and in any or all challenges. We will compare solutions where only IrRNA-seq data was used and solutions that include additional publicly-available data. Depending on the challenge, they will submit either a GTF or quantification file, additional metadata, and a link to a repository (e.g., Github) where a working copy of the exact analysis pipeline used to generate their results can be downloaded. We expect to re-run analysis pipelines for well-performing submissions to help ensure reproducibility. The evaluation of the challenge will comprise both bioinformatics and experimental approaches. SQANTI3 (https://github.com/ConesaLab/SQANTI3) will be used to obtain transcript features and performance metrics that will be computed on the basis of SIRV-Set 4 spike-ins, simulated data, and a set of undisclosed, manually curated transcript models defined by GENCODE ${ }^{15}$. Human models will further be compared to histone modification ChIP-seq, open chromatin, CAGE, and poly(A)-seq results. Experimental validation will be performed on a select number of loci with either high agreement or disagreement between sequencing platforms or analysis pipelines. Evaluation scripts and experimental protocols will be publicly available in advance of submission deadlines (Data and code availability).

Methods

124 Additional details of all protocols for library preparation and sequencing can be found at the 125 ENCODE DCC and is linked to each dataset produced by LRGASP (Supplementary Table 1).

\section{Capping SIRVs}

127 Exogenous synthetic RNA references (spike-ins) are widely used to calibrate measurements in 128 RNA assays, but they lack the 7-Methylguanosine $\left(\mathrm{m}^{7} \mathrm{G}\right)$ cap structure that most natural eukaryotic RNA transcripts bear at their 5' end. This characteristic makes commercial spike-in mixes unsuitable for library preparation protocols involving 5' cap enrichment steps. Therefore, we enzymatically added the appropriate $\mathrm{m}^{7} \mathrm{G}$ structure to the SIRV standards used in this challenge. Specifically, the pp5'N structure present at the 5' end of spike-in sequence was used as a template for the Vaccinia capping enzyme (catalog num M2080S, New England BioLabs) to add the $\mathrm{m}^{7} \mathrm{G}$ structure to SIRV-Set 4 (Iso Mix E0 / ERCC / Long SIRVs, catalog num 141.03, 
135 Lexogen). A total of ten vials of SIRV-Set $4(100 \mu \mathrm{l})$ were employed to perform the capping 136 reaction (final total mass of $535 \mathrm{ng}$ ). The reaction was performed following the

137 recommendations of the manufacturer's capping protocol with two minor changes: $3.5 \mu l$ of 138 RNAse inhibitors (RNasin Plus RNase Inhibitor, catalog num N2611, Promega) were added to 139 the capping reaction to avoid RNAse degradation, and the incubation time was extended from 14030 minutes to two hours, following a recommendation from New England BioLabs technical 141 support scientists. The final capping reaction was purified by using 1.8x AMPure RNA Clean XP 142 beads (catalog num. A63987, Beckman Coulter) and resuspended in $100 \mu$ of nuclease-free 143 water.

\section{Mouse and human RNA sample preparation}

145 Prior to distribution of biosample total RNA aliquots to each of the participating labs, $110 \mu \mathrm{g}$ of each biosample total RNA was spiked with Lexogen Long SIRV Set-4 quantification standards (catalog \# 141.03) at approximately $3 \%$ of the estimated mRNA mass present $(\sim 1 \%$ of total RNA). The mass of capped SIRVs used was $29.5 \mathrm{ng}$ and the mass of uncapped SIRVS used was $28.9 \mathrm{ng}$. In the case of direct RNA sequencing of one replicate of WTC-11 (ENCODE library accession ENCLB926JPE) and one replicate of mouse ES cells (ENCODE library accession ENCLB386NNT), only uncapped SIRV 4.0 were spiked in at approximately $3 \%$ of the estimated mass. Appropriate volumes of the spiked total RNA mixture to meet the input mass requirements for each library preparation method were then aliquoted separately, stored at -80 $\mathrm{C}$, and shipped on dry ice to participating labs.

\section{Manatee RNA sample preparation}

157 Blood samples from Florida manatees were collected during health assessments by the U.S 158 Geological Survey (USGS) Sirenia Project, the Florida Fish and Wildlife Conservation 159 Commission (FWC), and the University of Florida under U.S. Fish and Wildlife Service 160 (USFWS) permit \# MA791721-5 in Crystal River (Citrus County, Florida, USA) and in Satellite 161 Beach (Brevard County, Florida, USA) in December and January of 2018 and 2019 162 respectively. Samples were processed under the University of Florida USFWS permit 163 \#MA067116-2 following a protocol approved by the ethics committee (IACUC \# 201609674 \& 164 IACUC \# 201909674). Whole blood from minimally restrained Florida manatees were collected 165 from the medial interosseous space between the ulna and radio from the pectoral flippers. 166 Samples were drawn using Sodium Heparin 10-mL BD vacutainers (BD BioScience, New 
Jersey, U.S.A). Blood samples were spun on-site and the plasma was aliquoted, stored in liquid nitrogen or ice, and transferred to $-80^{\circ} \mathrm{C}$ once in the lab. The buffy coat (white blood cells) was flash-frozen in liquid nitrogen on-site and total RNA was extracted subsequently in the lab using STAT 60 (Tel-test Friendswood, TX) reagent. Approximately $350 \mu \mathrm{L}$ of the frozen buffy coat was added to $1 \mathrm{ml}$ of STAT 60 and vortexed for 30 seconds, $250 \mu \mathrm{L}$ of chloroform was added and the tube was centrifuged $20,800 \times \mathrm{g}$ for 15 minutes at $4^{\circ} \mathrm{C}$, to extract the RNA. This step was repeated and then RNA was precipitated from the supernatants overnight at $-20^{\circ} \mathrm{C}$ by the addition of $700 \mu \mathrm{L}$ isopropanol with $1.5 \mu \mathrm{L}$ of GlycoBlueTM $(15 \mathrm{mg} / \mathrm{mL}$ ) (Ambion, Invitrogen, Austin, TX) as a coprecipitant. Following centrifugation at $20,800 \times \mathrm{g}$ for 45 minutes, the pellet was washed with ethanol $70 \%$, air-dried, and resuspended in $20 \mathrm{~mL}$ of RNA secure (Ambion, Austin, TX). A DNAse treatment was performed using Turbo DNA-freeTM kit (Ambion, Austin, TX). A total of nine good-quality RNA samples were selected to create an RNA pool. These samples included 6 females, one calf, one lactating female and one male and had RIN values from 8.0 to 8.8 .

\section{Manatee genome sample preparation}

183 The genome of the Florida manatee Lorelei was sequenced using Nanopore and Pacbio. Lorelei is the same individual manatee for which an Illumina-based genome assembly was released by the Broad Institute in $2012^{16}$. An EDTA, $-80^{\circ} \mathrm{C}$ whole blood sample aliquot was used. gDNA was extracted from $1400 \mu$ of blood using the DNeasy kit (QIAGEN, MD, USA) following the companies' specifications for $100 \mu$ aliquots of blood. Thawed blood was diluted 1:1 with RNA free Phosphate buffered saline 1x (Gibco, UK), $20 \mu \mathrm{l}$ of proteinase K (QIAGEN, MD, USA), and 200 ul of AL lysis buffer (QIAGEN, MD, USA) and vortexed immediately. It was incubated at $56{ }^{\circ} \mathrm{C}$ for 10 minutes. Then, we added $200 \mu \mathrm{l}$ of ethanol $96 \%$ and mixed it thoroughly. The mixture was added to the DNeasy mini spin-column and centrifuged at 6,000 $\mathrm{x}$ $\mathrm{g}$ for 1 minute. The column was washed with $500 \mu$ of AW1 solution (QIAGEN, MD, USA) and centrifuged at 6,000 $\mathrm{xg}$ for 1 minute and followed with a wash with $500 \mu \mathrm{l}$ AW2 (QIAGEN, MD, USA ) and centrifuged $20,000 \times \mathrm{g}$ for 3 minutes. gDNA was eluted twice with $100 \mu \mathrm{l}$ of AE buffer added to the center of the column, incubated for 1 minute, and centrifuged $6,000 \times \mathrm{g}$ for 1 minute. The first and second elution from the DNeasy mini spin-column were pooled and concentrated using a speed vacuum for 20 minutes in which each preparation was reduced from 200 to $50 \mu \mathrm{l}$. All gDNA tubes were pooled and the DNA was cleaned with AM Pure magnetic beads (Beckman Coulter-Life Sciences, IN, USA) at a ratio of 0.5:1, beads volume to 
gDNA volume ( $50 \mu$ l of beads to $100 \mu$ l of gDNA). gDNA bound to the beads was washed twice with $1 \mathrm{ml}$ of $70 \%$ ethanol. Ethanol traces were removed by quick spin to the bottom of the tube and removed with a pipette. Then, the beads were dried for 2 minutes and gDNA was eluded in $55 \mu$ of EB buffer (QIAGEN, MD, USA) at $37^{\circ} \mathrm{C}$ with 10 minutes of incubation. This process was repeated twice. Quantification of gDNA was performed with a QubitTM fluorometer (Thermo Fisher Scientific) and the quality of the gDNA was assessed using a Genomic Agilent TapeStation (Santa Clara, CA, USA). The final DNA quantity was $28.8 \mu \mathrm{g}$ of DNA at a concentration of $267 \mathrm{ng} / \mathrm{\mu l}$. The DNA Integrity Number (DIN) was 8.8 and the peak size was $54.5 \mathrm{~kb}$.

PacBio cDNA synthesis was performed using a modified version of the Picelli protocol ${ }^{17}$ with the Maxima $\mathrm{H}$ - reverse transcriptase. Total RNA was treated with exonuclease to remove transcripts without a cap. $2 \mu \mathrm{l}$ of exonuclease-treated RNA were mixed with a priming reaction (RNAse inhibitor, dNTP's and water)was incubated at $72^{\circ} \mathrm{C}$ for 3 minutes, then ramps down to $50^{\circ} \mathrm{C}$. While in the PCR block we added oligo dT (stock concentration $10 \mathrm{nM}$ ) and were incubated $3 \mathrm{~min}$ at $50^{\circ} \mathrm{C}$. We then added a first strand synthesis buffer (5x RT buffer, TSOligo, water) that had previously been incubated at $50^{\circ} \mathrm{C}$ for one minute. The previous reaction was then incubated in the PCR block (Extension at $50^{\circ} \mathrm{C}$ for $90 \mathrm{~min}, 85^{\circ} \mathrm{C}$ for $5 \mathrm{~min}$ and held at $4^{\circ} \mathrm{C}$ ). To the same reaction we added a mix for amplification (2x reaction buffer, IS primers - $20 \mathrm{nM}$ stock, water and SeqAmp polymerase). Then we ran a PCR program to amplify the cDNA $\left(95^{\circ} \mathrm{C}\right.$ $1 \mathrm{~min}, 98^{\circ} \mathrm{C} 15 \mathrm{sec}, 65^{\circ} \mathrm{C} 30 \mathrm{sec}$ and $68^{\circ} \mathrm{C} 13 \mathrm{~min}$. The cycle repeats 10 times, which is followed by incubation at $72^{\circ} \mathrm{C}$ for $10 \mathrm{~min}$ and holding at $4^{\circ} \mathrm{C}$. The amplified products were purified using SPRI beads and checked for quality in a bioanalyzer.

\section{PacBio library preparation of human and mouse libraries}

226 To build PacBio libraries, we started from $500 \mathrm{ng}$ of polyA selected cDNA. The ends of the 227 cDNA were repaired first in order for the cDNA molecule to be suitable for ligation of SMRTbell 228 adapters. We added a damage repair reaction (DNA prep buffer, NAD and DNA damage repair) 229 and then incubated at $37^{\circ} \mathrm{C}$ for $30 \mathrm{~min}$. Then End prep mix was added and incubated at $20^{\circ} \mathrm{C}$ for $23030 \mathrm{~min}$ and $65^{\circ} \mathrm{C} 20 \mathrm{~min}$. Ligation of the adapter at the ends of the CDNA was done by adding a 231 ligation mix (pacbio adapters, ligation mix, ligation enhancer and ligation additive), then it was 
232 incubated at $20^{\circ} \mathrm{C}$ for $60 \mathrm{~min}$. Final libraries were cleaned up using SPRI beads and we

233 recorded the size and concentration of samples. Once the ligation step was done and the

234 libraries passed the QC, a sequencing primer was annealed to the adapters in the UCI GHTF

235 sequencing facility to allow for the binding of the polymerase during sequencing.

\section{CapTrap preparation for PacBio and ONT sequencing of human and mouse}

238 CapTrap is a technique developed by the Guigó laboratory (CRG, Barcelona, Spain) in 239 collaboration with the group of Piero Carninci in RIKEN, Japan. The method enriches for fulllength transcripts by selection of the 7-Methylguanosine $(\mathrm{m} 7 \mathrm{G})$ cap structure present at the 5' ends of RNA transcripts, followed by specific cap- and polyA- dependent linker ligations. The cDNA libraries generated using this method are compatible with long-read sequencing platforms (ONT or PacBio). The protocol starts with first strand synthesis (PrimeScript II Reverse Transcriptase, catalog num. 2690A, Takara) where $5 \mu \mathrm{g}$ of total RNA polyA+ RNAs are fully reverse transcribed using a 16-mer anchored dT oligonucleotide. First strand synthesis was performed at $42^{\circ} \mathrm{C}$ for 60 minutes. Resulting products were purified with 1.8x AMPure RNA Clean XP beads (catalog num. A63987, Beckman Coulter). After the first-strand generation, the m7G cap structure at the $5^{\prime}$ end of the transcripts is selectively captured using the CAP-trapper technique ${ }^{14,18}$, which leads to the removal of uncapped RNAs. The diol group on the $\mathrm{m}^{7} \mathrm{G}$ cap is oxidized with 1M NaOAc (pH 4.5) and NalO4 (250 mM). Tris $\mathrm{HCl}$ (1M, pH 8.5) was added to stop the reaction and the whole reaction was purified with 1.8x AMPure RNA Clean XP beads. Aldehyde groups were biotinylated using a mixture containing $\mathrm{NaOAc}(1 \mathrm{M}, \mathrm{pH} 6.0)$ and Biotin (Long Arm) Hydrazide (100 mM, catalog num. SP-1100, Vector Laboratories). The resulting mixture was then incubated for 30 minutes at $40^{\circ} \mathrm{C}$ and purified with 1.8x AMPure RNA Clean XP beads. Single strand RNA was degraded by RNase ONE Ribonuclease (catalog num. M4261, Promega) for 30 minutes at $37^{\circ} \mathrm{C}$ and purified with $1.8 x$ AMPure RNA Clean XP beads. The $\mathrm{m} 7 \mathrm{G}$ cap structure bound to biotin is then selected using M-270 streptavidin magnetic beads (catalog num. 65305, Thermo Fisher Scientific). M-270 streptavidin magnetic beads were equilibrated with

259 CapTrap Lithium chloride/Tween 20 based binding buffer. Sample recovered after RNase ONE 260 purification was bound to equilibrated M-270 streptavidin magnetic beads (incubation at $37^{\circ} \mathrm{C}$ for 26115 minutes), washed 3 times with CapTrap Tween20 based washing buffer and released by heat 262 shock for 5 minutes at $95^{\circ} \mathrm{C}$ and quickly cooled on ice. A second release was performed, and the 263 supernatant was also collected and mixed with the eluate from the previous release. The released 264 sample was treated with RNase H $(60 \mathrm{U} / \mu \mathrm{l}$, Ribonuclease $\mathrm{H}<$ RNase $H>$, catalog num. 2150, 
Takara), RNase ONE (10 U/Ml) and CapTrap release buffer (incubated at $37^{\circ} \mathrm{C}$ for 30 minutes), purified with 1.8x AMPure XP beads (catalog num. A63881, Beckman Coulter) and concentrated by using a speed vac. After this cap specific selection, two double-stranded linkers, carrying a unique molecular identifier (UMI), are specifically ligated to the first strand cDNA ${ }^{19}$. Linker ligation (DNA Ligation Kit <Mighty Mix>, catalog num. 6023, Takara) was performed in two separate steps. First the $5^{\prime}$ linker was ligated, purified twice, to completely eliminate the non-incorporated linkers, with 1.8x AMPure XP beads and concentrated by using a speed vac. Then the 3 ' linker was ligated, purified once with 1.8x AMPure XP beads and finally concentrated by using a speed vac. The double stranded linkers are converted into single strand by Shrimp Alkaline Phosphatase

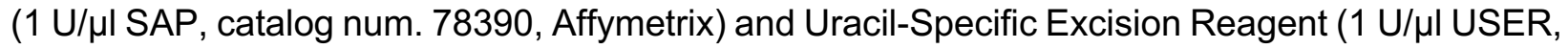
catalog num. M5505L, NEB) treatment. This reaction was incubated for 30 minutes at $37^{\circ} \mathrm{C}, 5$ minutes at $95^{\circ} \mathrm{C}$ and finally placed on ice. The sample was then purified with $1.8 x$ AMPure XP beads. After this treatment, the two linkers which serve as priming sites for the polymerase (2x HiFi KAPA mix, catalog num. 7958927001-KK2601, Kapa), enable the synthesis of the full-length second strand. The mixture was incubated for 5 minutes at $95^{\circ} \mathrm{C}, 5$ minutes at $55^{\circ} \mathrm{C}, 30$ minutes at $72^{\circ} \mathrm{C}$ and finally held at $4^{\circ} \mathrm{C}$ until $1 \mu \mathrm{l}$ Exonuclease I (20U/ $\mu \mathrm{l}$, catalog num. M0293S, NEB) was added to each sample. The sample was then incubated for 30 minutes at $37^{\circ} \mathrm{C}$ and afterwards, purified twice with $1.8 \mathrm{x}$ and $1.4 \mathrm{x}$ (respectively) AMPure XP beads and finally concentrated in a speed vac. The resulting cDNA is amplified (TaKaRa LA Taq, catalog num. RR002M, Takara) via long and accurate PCR (LA PCR) protocol. In order to avoid PCR duplicates, each sample was split in two PCR independent reactions and amplified 16 cycles with 15 seconds at $55^{\circ} \mathrm{C}$ for annealing, and 8 minutes at $65^{\circ} \mathrm{C}$ for extension. The 2 PCR replicates were merged and purified with 1x AMPure XP beads. Samples were quantified with Qubit (Qubit 4 Fluorometer, Thermo Fisher Scientific) and quality-checked with BioAnalyzer (Agilent 2100 Bioanalyzer, Agilent Technologies).

CapTrap MinION cDNA sequencing was performed with $500 \mathrm{ng}$ of cDNA sample coming from CapTrap cDNA protocol and strictly following the SQK-LSK109 adapter ligation protocol (ONT). The cDNA sequencing on MinION platform was performed using ONT R9.4 flow cells and the 294 standard MiniKNOW protocol. 


\section{R2C2 preparation for ONT sequencing of human and mouse}

300 For each biological replicate, two libraries were created, a regular (non-size selected), and a size selected library of cDNA over $2 \mathrm{~kb}$ in length to achieve higher coverage of longer transcripts. For each RNA sample, $400 \mathrm{ng}$ was used to generate full-length single stranded cDNA using an indexed oligo(dT) primer and a template switching oligo (TSO). PCR was used to generate the second strand and amplify the library. The CDNA was then isolated by SPRI bead clean up. For the size selected libraries, cDNA was run on a $1 \%$ low melt agarose gel. A smear in the range of $2-10 \mathrm{~kb}$ was excised from the gel and digested with beta-agarase followed by SPRI bead clean up. At this point, indexed cDNA from each biological replicate was pooled together equally. cDNA was circularized using a short DNA splint with sequence complementary to the cDNA ends by Gibson Assembly (NEBuilder, NEB) with a 1:1 cDNA:splint ratio (100 ng each). After Gibson assembly, a linear digestion (Exol, Exolll, and Lambda Exonuclease) was performed to eliminate non-circularized DNA. The circular Gibson assembly product was cleaned up using SPRI beads. The circularized library was used as template for rolling circle amplification (RCA) using Phi29 polymerase and random hexamer primers. Following the RCA reaction, T7 endonuclease was used to debranch the DNA product. A DNA clean and concentrator column was used to purify the DNA. Purified RCA product was sizeselected using a $1 \%$ low melt agarose gel. The main band just over the $10 \mathrm{~kb}$ marker was excised from the gel and digested with beta-agarase followed by SPRI bead clean up. The cleaned and size selected RCA product was sequenced using the ONT 1D Genomic DNA by Ligation sample prep kit (SQK-LSK109) and MinION flow cells (R9.4.1) following the manufacturer's protocol. Flow cells were nuclease flushed and reloaded with additional library following ONT Nuclease Flush protocol.

\section{cDNA preparation for ONT sequencing of human and mouse}

324 Library preparation was done from total RNA (200ng) using SQK-PCS110 kit from ONT for 325 PCR-cDNA sequencing. Briefly, CDNA RT adapters were annealed and ligated to full length 326 RNAs using NEBNext® Quick Ligation Reaction Buffer (NEB B6058) and T4 DNA Ligase (NEB 327 M0202). Bead clean up was done using Agencourt RNAClean XP beads. Purified RNA with 328 CRTA top strand, RT primers, and dNTPs (NEB N0447) were incubated at RT for 15 mins to 329 generate primer-annealed RNA. Reverse transcription and strand-switching was performed with 330 Maxima $\mathrm{H}$ Minus RT enzyme in presence of strand-switching primers at $42^{\circ} \mathrm{C}$ for 90 mins 331 followed by heat inactivation at $85^{\circ} \mathrm{C}$ for 5 mins. Reverse transcribed samples were PCR 
332 amplified using cDNA primers and LongAmp Hot Start Master Mix (NEB, M0533S). Samples

333 were treated with NEB exonuclease I (NEB, M0293) for 15 mins at $37^{\circ} \mathrm{C}$ to degrade linear

334 single-stranded DNA, followed by enzyme inactivation at $80^{\circ} \mathrm{C}$ for 15 mins. Samples were

335 purified with Agencourt AMPure XP beads. Elution was done with 12 ul of elution buffer. 1ul of

336 libraries was electrophoresed on TapeStation screentapes to assess size distribution, quantity

337 and quality of library. FLO-MIN106D flow cells were primed with EXP-FLP002 kit reagents

338 followed by loading of PCR-cDNA library mixed with rapid adapter F (along with sequencing

339 buffer and loading beads). Sequencing of the library was performed without any size selection

340 using MinION Mk1B devices and MinKNOW software interface.

dRNA preparation for ONT sequencing of human and mouse

dRNA libraries were prepared from 75ug total RNA. RNA samples were poly-A selected using the NEXTFLEX poly-A kit. Purified mRNA was eluted in 12uL NF H2O. Library preparation was performed on purified mRNA using the SQK-RNA002 kit. Direct RNA RT adapters were annealed and ligated to full-length mRNA using T4 DNA Ligase, NEBNext Quick Ligation

347 Reaction Buffer, and Nanopore's RNA CS. Adapter-ligated mRNA was incubated with dNTPs,

$3485 x$ first-strand buffer, nuclease-free water, SuperScript IV, and 0.1M DTT to create a cDNA-RNA 349 hybrid. This reverse-transcription (RT) step is recommended by Nanopore to reduce secondary structure formation of the mRNA as it is being sequenced. RTed RNA was purified using RNAClean XP beads. Nanopore adapters were ligated onto the RTed RNA using NEBNext Quick Ligation Reaction Buffer and T4 DNA Ligase. Following RNAClean XP bead cleanup, the libraries were eluted in 21uL of Nanopore's Elution Buffer. $1 \mathrm{uL}$ of each library was quantified on the TapeStation to ensure nucleic acid concentration was at minimum $\sim 200 \mathrm{ng}$. Libraries were loaded into MinION flow cells using the EXP-FLP002 Flow Cell Priming Kit. Libraries were sequenced for 72 hour runs.

\section{Manatee ONT genome sequencing}

$3592 \mu \mathrm{g}$ of genomic DNA in a total volume of $100 \mu \mathrm{l}$ was fragmented with the g-Tube fragmentation method (Covaris, Woburn, MA, USA) by using centrifugation at 6,000x $\mathrm{g}$ for $1 \mathrm{~min}$. The large DNA fragments were enriched by using 0.85x volume of Agencourt AMPure XP beads

362 (Beckman Coulter, Brea, CA, USA) in the purification procedure. The enriched DNA fragments 363 were subjected to library preparation with Nanopore Genomic DNA Ligation Sequencing Kit 
364 (Oxford Nanopore Technologies, Oxford, UK) following the manufacture's protocol. A total of $700 \mathrm{ng}$ of final library product was loaded on a flow cell and sequenced with a Nanopore GridION sequencer (Oxford Nanopore Technologies, Oxford, UK) for a 72-hr run. A total of 5 flow-cell runs were conducted for this project.

\section{Manatee cDNA Pacbio library preparation and sequencing}

369 Approximately $280 \mathrm{ng}$ of total pooled RNA were processed according to a modified IsoSeq protocol. The sample was spiked-in with the uncapped E2 RNA variant control mix (SIRVs, Lexogen, Cat \# 025.03 ) at a $2.83 \%$ mass proportion relative to the total RNA. The resulting mixture was subjected to a globin removal step using the QIAseq FastSelectTM- HRM Globin removal reagent (cat \# 334376). This kit was designed for globin removal from human, mouse, and rat tissues and was found to perform with various degrees of efficiency on blood from a wide variety of samples of mammalian origin. Globin removal was performed as recommended in the QIAseq FastSelectTM- -rRNA HRM -Globin Handbook (Oct 2019) in the NEBNext Ultra II section, except that the high-temperature fragmentation step was omitted. The globin removal reaction (9 $\mu \mathrm{l}$ ) contained: $280 \mathrm{ng}$ sample (RNA plus 2.83\% SIRVs), QIAseq FastSelect globin removal reagent, $2 \mu$ l NEBNext Single Cell RT Primer Mix (NEB \#6421), and $2.25 \mu$ of NEBNext Single Cell RT buffer (4x). This mixture was prepared in a $0.2 \mathrm{ml} \mathrm{PCR}$ tube and subjected to a stepwise series of 2 min incubations each of $75^{\circ} \mathrm{C}, 70^{\circ} \mathrm{C}, 65^{\circ} \mathrm{C}, 60^{\circ} \mathrm{C}, 55^{\circ} \mathrm{C}, 37^{\circ} \mathrm{C}$ and $25^{\circ} \mathrm{C}$. At this point, the sample was snap-cooled by transferring to a pre-chilled freezer block until ready for the RT and amplification steps. From this point on, cDNA synthesis was done as described in the "Protocol for Low Input RNA: cDNA Synthesis and Amplification" (NEB \#E6421) starting on section 2.3. More specifically, the template "RT and Template Switching" reaction consisted of $9 \mu$ l of globin-removed RNA, $2.75 \mu$ l NEBNext Single Cell RT Buffer (4x), 1 $\mu \mathrm{l}$ of NEBNext Template Switching Oligo, $2 \mu \mathrm{l}$ of NEBNext Single Cell RT Enzyme Mix and enough water to bring the total to $20 \mu$ l. The reaction was incubated in a thermocycler for $90 \mathrm{~min}$ at $42{ }^{\circ} \mathrm{C}$ and $10 \mathrm{~min}$ at $72{ }^{\circ} \mathrm{C}$. The cDNA products were split into four aliquots for PCR amplification $(100 \mu \mathrm{l})$ reactions containing $2 \mu \mathrm{l}$ NEBNext Single Cell cDNA PCR Primer, $0.5 \mu l$ 10X NEBNext Cell Lysis Buffer, $50 \mu \mathrm{l}$ NEBNext Single Cell cDNA PCR Master Mix, $5 \mu \mathrm{l}$ RT and

392 Template Switching reaction and water. Amplified cDNA was purified by AMPure, one round at 3930.8 to 1.0 beads to sample ratio and one round at $0.65: 1.0$ ratio. The yield of amplified cDNA by 394 this modified protocol (300-400 ng) was about 10-fold lower than the standard protocol (i.e., 395 without globin-removal). The average cDNA size was $\sim 1400 \mathrm{bp}$. When increased amounts of 396 cDNA were desired the cDNA was amplified by 5 additional PCR cycles. 
Two preps obtained with the above described protocol were pooled together and $500 \mathrm{ng}$ were loaded on an electrophoretic lateral fractionation system (ELF, SageScience). Fragments above $2.5 \mathrm{~kb}$ were collected, re-amplified ( 10 cycles), and re-pooled equimolarly with non-sizeselected cDNA fragments. This re-pooled cDNA prep is referred to as "enriched cDNA_>2.5kb". Both non_enriched cDNA and enriched cDNA_>2.5kb cDNA were used for SMRT bell library was constructed starting with $1 \mu \mathrm{g}$ of cDNA as described (PacBio IsoSeq protocol 101-070-200 Version 06, September 2018). Briefly, SMRTbell adaptors (Iso-SeqTM) were added using reagents from the PacBio SMRTbell Template Prep Kit 1.0-SPv3 starting with either $200 \mathrm{ng}$ (for enriched cDNA $>2.5 \mathrm{~kb}$ ) or $700 \mathrm{ng}$ (for non enriched cDNA). The main steps included: DNA Damage Repair, End Repair, Blunt-end ligation of SMRT bell adaptors, and ExollI/ExoVII treatment. This procedure resulted in $\sim 25-30 \%$ yield. Finally, libraries were eluted in 15 ul of 10 $\mathrm{nM}$ Tris $\mathrm{HCl}, \mathrm{pH}$ 8.0. Library fragment size was estimated by the Agilent TapeStation (genomic DNA tapes), and this data was used for calculating molar concentrations.

The enriched cDNA $>2.5 \mathrm{~kb}$ library was diffusion-loaded on a single SEQUEL SMRT cell (University of Florida, ICBR-NGS core lab) at loading concentration was 10 pM, using 4-hr preextension, $20 \mathrm{hr}$ movies and $\mathrm{v} 3$ chemistry reagents (for binding and sequencing). All other steps for sequencing were done according to the recommended protocol by the PacBio SMRT Link Sample Setup and Run Design modules (SMRT Link 6.0).

415 The non enriched cDNA library loaded on three Sequel II SMRT cells at University of California, 416 Irvine.

\section{Manatee cDNA Nanopore library preparation and sequencing}

418 One hundred and fifty nanograms of total pooled RNA were processed according to a modified 419 ONT cDNA-PCR Sequencing protocol (cDNA-PCR-PCS109, version PCS_9085 v109 revJ Aug 14, 2019). Spike-in and globin depletion treatment was conducted as described for Pacbio library preparation. In this case, the globin removal reaction (11 ul) contained: sample (RNA plus SIRVs), globin removal reagent, $1 \mathrm{mM}$ dNTP, $0.2 \mu \mathrm{M}$ VPN primer from the Nanopore cDNA synthesis protocol (i.e., in place of random primers), and 1X RT buffer (ThermoFisher). This mixture was prepared in a $0.2 \mathrm{ml} \mathrm{PCR}$ tube and submitted to a stepwise series of 2 min incubation for each of $75^{\circ} \mathrm{C}, 70^{\circ} \mathrm{C}, 65^{\circ} \mathrm{C}, 60^{\circ} \mathrm{C}, 55^{\circ} \mathrm{C}, 37^{\circ} \mathrm{C}$ and $25^{\circ} \mathrm{C}$. At this point, the sample was snap-cooled by transferring to a pre-chilled freezer block until ready for the RT and amplification steps. From this point on, cDNA synthesis was done as described in the cDNA-PCR Sequencing (SQK-PCS109) Nanopore manual starting on page 9 (Version: PCS_90985_v109_revJ_14Aug2019). A single 
430 This process resulted in approximately 2 micrograms of "full-length" cDNA with an average size 431 of $\sim 1800 \mathrm{bp}$. One size-selected library was constructed by loading $1500 \mathrm{ng}$ of this cDNA on an 432 electrophoretic lateral fractionation system (ELF, SageScience), collecting.5 kb were collected, 433 re-ampliying (6 cycles) and re-pooling with non-size-selected cDNA fragments. Adaptor ligation 434 and sequencing were performed according to the cDNA-PCR Sequencing (SQK-PCS109) 435 Nanopore manual. Between 120-140 fmol of cDNA was loaded on a FLO-MIN106D (R9.4 436 SpotON) flow cell for sequencing on the minION device. Two runs were done on non-size437 selected manatee cDNA, while only one run was done on the cDNA that had been enriched with $438>2.5 \mathrm{~kb}$ fragments. Sequencing runs were allowed to proceed for 48 hours.

\section{Long-read data processing}

441 Basecalling of ONT data from human, mouse and manatee was performed with Guppy 4.2.2

442 and hac 9.4.1 config file, with default parameters, except: --qscore_filtering --min_qscore 7

443 (these non-default parameters were used in all ONT cDNA runs except for R2C2 datasets).

444 Direct RNA basecalling was also performed with Guppy 4.4.2 with the following configurations: -

445 -qscore_filtering yes --min_qscore 7 --reverse_sequence yes

446 --u_substitution yes

448 PacBio full-length non-chimeric (FLNC) reads were generated with CCS 4.2 .0 (parameters: -449 noPolish --minLength=10 --minPasses=3 --min-rq=0.9 --min-snr=2.5), Lima 1.11.0 (parameters:

450 FASTA with the appropriate adapters --isoseq --min-score 0 --min-end-score 0 --min-signal451 increase 10 --min-score-lead 0), and Refine 3.3.0 (parameters: --min-polya-length 20 --require452 polya).

454 Consensus R2C2 reads were generated with C3POa v1.0.0

455 (https://github.com/rvolden/C3POa/tree/gonk) with default options

457 Sequence data are provided in FASTQ format. For PacBio data, subreads are provided in 458 unaligned BAM format and for R2C2 data, subreads are provided in FASTQ (Supplementary 459 Table 1). 


\section{Reference genome and annotations}

461 For submissions of transcript models and quantification, transcript annotations and genome 462 models corresponding to GENCODE human v38 and mouse M27 will be used. Submissions of 463 challenge predictions are expected to end in Fall 2021, prior to the release of GENCODE 464 human v39 and mouse M28. The newly released GENCODE annotations will, therefore, be 465 used for the evaluations. GRCh38 is the reference genome sequence for human and GRCm39 466 for mouse, GENCODE annotations are based on these genomes. Please note that GENCODE 467 M25 and earlier annotation releases are based on GRCm38.

\section{Simulated data}

470 Simulating RNA reads simply from the reference transcriptome would only allow the assessment reconstruction of known transcript models. Thus, we extended both human and mouse annotations with artificial novel transcripts. To obtain those, we mapped reference transcripts of an undisclosed mammalian organism to the human and mouse genomes and converted the alignments into transcript models using SQANTI ${ }^{20}$. We then arbitrarily selected isoforms of known genes that have only canonical splice sites (GT-AG, GC-AG and AT-AC) and merged them into human and mouse GENCODE Basic annotations.

To generate realistic isoform expression profiles we selected undisclosed human and mouse long read datasets and quantified them simply by mapping to the reference transcripts with minimap2 v2.17 (Li, 2018). Artificial novel isoforms were assigned arbitrary expression values. Generated expression profile was further used for simulating short and long reads.

To simulate reads produced by different sequencing platforms we used existing simulation methods. Illumina $2 \times 150 \mathrm{bp}$ read pairs were generated with the RSEM simulator ${ }^{21}$ using an error model obtained from real RNA-Seq data ${ }^{22}$ ( accession number ERR1474891). ONT reads were simulated with $\mathrm{NanoSim}^{23}$ using pre-trained cDNA and dRNA models available in the package with average error rate of $15.9 \%$ (4.8\% substitutions, $6.0 \%$ deletions, $5.1 \%$ insertions) and $11.2 \%$ (2.8\% substitutions, $5.9 \%$ deletions, $2.5 \%$ insertions) respectively. PacBio CCS reads were obtained with IsoSeqSim (https://github.com/yunhaowang/lsoSeqSim), which

490 truncates input reference transcript sequences and uniformly inserts errors according to given 491 probabilities. We used Sequel II truncation probabilities provided along with the package. Error 492 rate was estimated using real PacBio cDNA CCS reads obtained in this work as $1.6 \%(0.4 \%$ 
substitutions, $0.6 \%$ deletions, $0.6 \%$ insertions). Additionally, polyA tails were attached to the 3 ' end of reference transcript sequences prior to running the simulation.

We simulated two datasets containing reads from all 3 platforms listed above but with slightly different properties. Human datasets were simulated with 100 million Illumina read pairs, 30 million ONT cDNA and 10 million PacBio reads. Mouse datasets also contained 100 million Illumina read pairs, but equal amounts of PacBio CCS and ONT dRNA reads were generated (20 million sequences each).

To allow users to simulate their own data, the methods described above are implemented as simple command-line scripts which are available at https://github.com/LRGASP//rgaspsimulation/.

\section{CAGE data of WTC-11 samples for validation of transcript 5' ends}

507 CAGE data from WTC-11 samples are being produced for validation of transcript 5' ends;

508 therefore, will not be released until the close of the challenge submissions. CAGE data will be 509 obtained from two RNA biological replicates of WTC-11, from the same exact RNA used for 510 long-read sequencing.

512 The $15 \mu \mathrm{g}$ of WTC-11 RNAs from each biological replicate, ENCODE BioSample Accession 513 \#ENCBS944CBA and \#ENCBS474NOC, were used for the single strand (ss)CAGE library 514 preparation described in the published protocol ${ }^{24}$. Briefly, the $15 \mu \mathrm{g}$ RNAs were aliquoted to 5 $515 \mu \mathrm{g}$ in three tubes and reverse transcribed to cDNAs with random primers, and the RNA-cDNA 516 hybrids were cap-trapped by the streptavidin beads. The single strand cDNAs were released

517 from the beads and ligated to the Illumina adaptors with an index. 1080 amols of the cap-

518 trapped single strand cDNAs from each biological replicate were sequenced by Illumina HiSeq 519 Rapid SBS Kits v2 (SR, 150 cycles, 1 lane for each), producing approximately 40 million reads 520 per sample.

\section{QuantSeq of human and mouse samples for validation of transcript 3' ends}

523 QuantSeq data (3' end sequencing) from challenge 1 and 2 samples are being produced for 524 validation of 3' ends; therefore, this data will not be released until the close of the challenge 
525 submissions. Data will be obtained from two RNA biological replicates of WTC-11, from the

526 same exact RNA used for long-read sequencing.

\section{Full-length transcript validation with NRCeq}

529

Depending on sample availability, we may further sequence the WTC-11 cell line using NRCeq,

530 a method for direct RNA sequencing which can distinguish full length reads (i.e., dRNA reads

531 containing both the 5' cap and polyA tail) ${ }^{25}$. NRCeq uses an oligomer adaptation approach to

532 ligate an adapter specifically to 5' $\mathrm{m7G}$ capped RNAs and performs polyA-selected direct RNA

533 sequencing. NRCeq would provide additional data to validate start-to-end RNA transcript

534 sequence without RT-PCR artifacts ${ }^{26}$. Because the technique requires approximately $2.5 \mathrm{ug}$ of

535 poly(A)-selected RNA, the sequencing will be performed on two independent biological

536 replicates of WTC-11 that were not from the original cell batch from which long-read sequencing

537 was performed.

538

539 GENCODE benchmarks and computational evaluation

540 Full manual annotation will be undertaken on 50 selected loci on both the human and mouse

541 reference genomes. Transcript models will only be annotated during this exercise based on their

542 support from long transcriptomic datasets generated by the consortium specifically for LRGASP.

543 That is, no transcript annotation will be based on transcriptomic data from externally produced

544 datasets, although annotators will use any publicly available orthogonal data to aid interpretation

545 of aligned consortium data. For example, Fantom 5 CAGE datasets will be used to help identify

546 transcription start sites and transcript 5' ends and RNA-seq-supported introns derived from high

547 throughput reanalysis pipelines such as Recount will be used to support putative introns

548 identified in the alignments of long transcriptomic data.

550 Manual annotation will be performed according to the guidelines of the HAVANA (Human And

551 Vertebrate Analysis aNd Annotation) group ${ }^{15,27}$. Transcriptomic data will be aligned to the

552 human and mouse reference genome using appropriate methods. We will test the benefits of

553 aligning the transcriptomic data using multiple methods to reduce the impact of alignment errors 554 and artefacts. 
Annotators will also take advantage of local alignment tools integrated into annotation software to give further alternative views of alignments and improve annotation accuracy. Transcript models will be manually extrapolated from the alignments by annotators using the otter annotation interface ${ }^{28}$. Alignments will be navigated using the Blixem alignment viewer ${ }^{29,30}$ and where required visual inspection of the dot-plot output from the Dotter tool ${ }^{31}$ will be used to resolve any alignment with the genomic sequence that was unclear or absent from Blixem.

562 Short alignments ( $<15$ bases) that cannot be visualized using Dotter will be detected using 563 Zmap DNA Search ${ }^{31}$ (essentially a pattern matching tool). The construction of exon-intron 564 boundaries will require the presence of canonical splice sites (defined as GT-AG, GC-AG and 565 AT-AC) and any deviations from this rule will be given clear explanatory tags (for example non566 canonical splice site supported by evolutionary conservation). All non-redundant splicing 567 transcripts at an individual locus will be used to build transcript models, and all alternatively 568 spliced transcripts will be assigned an individual biotype based on their putative functional 569 potential. Once the correct transcript structure has been ascertained the protein-coding potential 570 of the transcript will be determined on the basis of its context within the locus, similarity to 571 known protein sequences, the sequences of orthologous and paralogous proteins, candidate 572 coding regions (CCRs) identified by PhyloCSF, evidence of translation from mass spectrometry 573 and Ribo-seq data, the presence of Pfam functional domains, the presence of possible 574 alternative ORFs, the presence of retained intronic sequence and the likely susceptibility of the 575 transcript to nonsense-mediated mRNA decay (NMD). Although the annotation of transcript 576 functional biotype and CDS is not required of submitters, it will be added to transcripts as a 577 matter of routine manual annotation and may be used to investigate the detection or non578 detection of groups of transcripts by submitters. Where necessary, annotations will be checked 579 by a second annotator to ensure completeness and consistency of annotation between the 580 genes annotated for LRGASP and the remainder of the EnsembI/GENCODE geneset.

\section{Computational evaluation of transcript isoform detection and quantification}

582 Challenge 1 Evaluation: Transcript isoform detection

583 Four sets of transcripts will be used for evaluation of transcript calls made on human and mouse 584 IrRNA-seq data

585 1. Lexogen SIRV-Set 4 (SIRV-Set 3 plus 15 new long SIRVs with sizes ranging from 4 to 586 $12 \mathrm{~kb})$ 
2. Comprehensive GENCODE annotation: human v39, mouse vM28. GENCODE human v28 and vM27 are available at the time of the LRGASP data release and new versions of GENCODE will be released after the close of LRGASP submissions.

3. A set of transcripts from a subset of undisclosed genes which will be manually curated by GENCODE. These transcripts will thus be considered high-quality models derived from LRGASP data

The rationale for including these different types of transcript data is that each set creates a different evaluation opportunity, but also has its particular limitations. For example, SIRVs and simulated data provide a clear ground truth that allows the calculation of standard performance metrics such as sensitivity, precision or false discovery rate. Evaluation of SIRVs can identify potential limitations of both library preparation as well as sequencing, but the SIRVs themselves represent a dataset of limited complexity. Higher complexity can be generated when simulating long reads based on actual sample data. However, read simulation algorithms only capture some potential biases of the sequencing technologies (e.g., error profiles) and not of the library preparation protocols. In any case, both types of data approximate, but do not fully recapitulate real-world datasets. Evaluation against the GENCODE annotation ${ }^{15}$ represents this real dataset scenario, although in this case the ground truth is not entirely known. This limitation will be partially mitigated by the identification of a subset of GENCODE transcript models that will be revised and deemed as high-confidence by GENCODE curators, and by follow-up experimental validation for a small set of transcripts using semi-quantitative RT-PCR and quantitative PCR (qPCR) approaches. In this way, although an exhaustive validation of the real data is not possible, estimates of the methods' performances can be inferred. By putting together evaluation results obtained with all these different benchmarking datasets, insights will be gained on the performance of the library preparation, sequencing and analysis approaches both in absolute and in relative terms. The evaluation of the transcript models will be guided by the use of SQANTI categories ${ }^{20}$ (Fig

617 incorporate additional definitions and performance metrics to provide a comprehensive

618 framework for transcript model assessment (Table 2). The evaluation considers the accuracy of 619 the transcript models both at splice junctions and at $3^{\prime} / 5^{\prime}$ transcript ends. It will take into 620 account external sources of evidence such as CAGE data, polyA annotation and support by 
621 Illumina reads (Fig 2b). A number of novel transcripts detected by all or most pipelines, as well

622 as pipeline-, platform-, or library- preparation specific transcripts will be selected for

623 experimental validation and manual review by the GENCODE project. The evaluation script is

624 provided to participants (Data and code availability).

Table 2: Transcript Classifications and Definitions used by the LRGASP computational evaluation

\begin{tabular}{|c|c|}
\hline Classification & Description \\
\hline Full Splice Match (FSM) & $\begin{array}{l}\text { Transcripts matching a reference transcript at all } \\
\text { splice junctions }\end{array}$ \\
\hline Incomplete Splice Match (ISM) & $\begin{array}{l}\text { Transcripts matching consecutive, but not all, } \\
\text { splice junctions of the reference transcripts }\end{array}$ \\
\hline Novel in Catalog (NIC) & $\begin{array}{l}\text { Transcripts containing new combinations of 1) } \\
\text { already annotated splice junctions, 2) novel splice } \\
\text { junctions formed from already annotated donors } \\
\text { and acceptors, or } 3 \text { ) unannotated intron retention }\end{array}$ \\
\hline Novel Not in Catalog (NNC) & Transcripts using novel donors and/or acceptors \\
\hline Reference Match (RM) & $\begin{array}{l}\text { FSM transcript with } 5^{\prime} \text { and } 3^{\prime} \text { ends within } 50 \text { nts of } \\
\text { the transcription start site (TSS)/transcription } \\
\text { termination site (TTS) annotation }\end{array}$ \\
\hline _3'_polyA_supported & $\begin{array}{l}\text { Transcript with polyA signal sequence support or } \\
\text { short-read 3' end sequencing (e.g. QuantSeq) } \\
\text { support at the 3'end }\end{array}$ \\
\hline -5'_CAGE_supported & Transcript with CAGE support at the 5 'end \\
\hline _3'_reference_supported & $\begin{array}{l}\text { Transcript with } 3 \text { 'end within } 50 \text { nts from a } \\
\text { reference transcript TTS }\end{array}$ \\
\hline _5'_reference_supported & Transcript with 5 'end within 50 nts from a \\
\hline
\end{tabular}




\begin{tabular}{|c|c|}
\hline & reference transcript TSS \\
\hline $\begin{array}{l}\text { Supported Reference Transcript Model } \\
\text { (SRTM) }\end{array}$ & $\begin{array}{l}\text { FSM/ISM transcript with } 5^{\prime} \text { end within } 50 \text { nts of the } \\
\text { TSS or has CAGE support AND } 3^{\prime} \text { end within } 50 \\
\text { nts of the TTS or has polyA signal sequence } \\
\text { support or short-read } 3^{\prime} \text { end sequencing support }\end{array}$ \\
\hline $\begin{array}{l}\text { Supported Novel Transcript Model } \\
\text { (SNTM) }\end{array}$ & $\begin{array}{l}\text { NIC/NNC transcript with } 5^{\prime} \text { end within } 50 \text { nts of the } \\
\text { TSS or CAGE support AND } 3^{\prime} \text { end within } 50 \text { nts of } \\
\text { the TTS or has polyA signal sequence support or } \\
\text { short-read } 3^{\prime} \text { end sequencing support AND } \\
\text { Illumina read support at novel junctions }\end{array}$ \\
\hline$\%$ Long Read Coverage (\%LRC) & $\begin{array}{l}\text { Fraction of the transcript model sequence length } \\
\text { mapped by one or more long reads }\end{array}$ \\
\hline Redundancy & \# LR transcript models / reference model \\
\hline $\begin{array}{l}\text { Longest Junction Chain } \\
\text { ISM } \\
\text { NIC / NNC }\end{array}$ & $\begin{array}{l}\text { \# junctions in ISM / \# junctions reference } \\
\text { \# reference junctions / \# junctions in NIC/NNC }\end{array}$ \\
\hline Intron retention (IR) level & Number of IR within the NIC category \\
\hline Illumina Splice Junction (SJ) Support & $\%$ SJ in transcript model with Illumina support \\
\hline Full Illumina Splice Junction Support & $\%$ transcripts in category with all SJ supported \\
\hline$\%$ Novel Junctions & \# of new junctions / total \# junctions \\
\hline$\%$ Non-canonical junctions & \# of non-canonical junctions / total \# junctions \\
\hline \% Non-canonical transcripts & $\begin{array}{l}\% \text { transcripts with at least one non-canonical } \\
\text { junction }\end{array}$ \\
\hline Intra-priming & Evidence of intra-priming (described in ${ }^{20}$ ) \\
\hline RT-switching & Evidence of RT-switching (described in ${ }^{20}$ ) \\
\hline
\end{tabular}


631 Given these definitions, evaluation metrics are specified for each type of data.

634 In order to evaluate SIRVs, we will extract from each submission all transcript models that 635 associate to SIRV sequences after SQANTI3 analysis. This not only includes FSM and ISM 636 isoforms of SIRVs, but also NIC, NNC, antisense and fusion transcripts mapping to SIRV loci.

637 The metrics for SIRV evaluation are defined as follows.

638

639 Table 3: Metrics and definitions for evaluation against SIRVs

\begin{tabular}{|l|l|}
\hline SIRV_transcripts & Transcripts mapping to a SIRV chromosome \\
\hline Reference SIRV (rSIRV) & Ground truth SIRV model \\
\hline True Positive detections (TP) & rSIRVs identified as RM \\
\hline Partial True Positive detections (PTP) & rSIRVs identified as ISM or FSM_non_RM \\
\hline False Negative (FN) & rSIRVs without FSM or ISM \\
\hline False Positive (FP) & NIC + NNC + antisense + fusion SIRV_transcripts \\
\hline Sensitivity & TP/rSIRVs \\
\hline Precision & RM/SIRV_transcripts \\
\hline Non_redundant Precision & TP/SIRV_transcripts \\
\hline Positive Detection Rate & unique(TP+PTP)/rSIRVs \\
\hline False Discovery Rate & (SIRV_transcripts - RM)/SIRV_transcripts \\
\hline Redundancy & (FSM + ISM)/unique(TP+PTP) \\
\hline
\end{tabular}

\section{Simulated Data}

643 The simulated data contains both transcript models based on the current GENCODE annotation

644 and a number of simulated novel transcripts that will result in true NIC and NNC annotations. 
645 Transcript models generated from simulated data will be analysed by SQANTI3 providing a GTF

646 file that includes all simulated transcripts (GENCODE and novel) and excludes all transcripts for

647 which reads were not simulated. The evaluation metrics for simulated data are defined as

648 follows:

649

650 Table 4: Metrics and definitions for evaluation against simulated data

\begin{tabular}{|c|c|}
\hline$P$ & All simulated transcripts \\
\hline True Positive (TP) & RM \\
\hline TP_ref & RM to GENCODE models \\
\hline TP_novel & RM to simulated novel transcript models \\
\hline Partial True Positive (PTP) & ISM or FSM_non_RM \\
\hline PTP_ref & ISM or FSM_non_RM of GENCODE models \\
\hline PTP_novel & ISM or FSM_non_RM of simulated novel models \\
\hline False Negative (FN) & Simulated transcripts without RM or PTP calls \\
\hline FN_ref & Simulated GENCODE models without RM or PTP calls \\
\hline FN_novel & Simulated novel models without RM or PTP calls \\
\hline False Positive (FP) & $\mathrm{NIC}+\mathrm{NNC}+$ antisense + fusion \\
\hline \multicolumn{2}{|l|}{ Sensitivity } \\
\hline Sens_ref & TP_ref/P(GENCODE) \\
\hline Sens_novel & TP_novel/P(Simulated novel) \\
\hline Precision & $T P /(T P+P T P+F P)$ \\
\hline Positive Detection Rate & $(\mathrm{TP}+\mathrm{PTP}) / \mathrm{P}$ \\
\hline False Discovery Rate & $(\mathrm{FP}+\mathrm{PTP}) /(\mathrm{TP}+\mathrm{PTP}+\mathrm{FP})$ \\
\hline Redundancy & \# FSM and ISM per simulated transcript model \\
\hline
\end{tabular}

651

652

653

Comprehensive GENCODE annotation 
654 Submitted transcript models will be analyzed with SQANTI3 using the newly released

655 GENCODE annotation and different metrics will be obtained for FSM, ISM, NIC, NNC and Other 656 models according to the scheme depicted below. Transcripts from new genes included in the 657 latest annotation release will be catalogued as "Intergenic" initially, but considered FSM, ISM,

658 NIC or NNC with an updated GENCODE annotation. This will allow evaluation of gene and 659 transcript discovery on unannotated regions.

Table 5: Metrics for evaluation against GENCODE annotation

\begin{tabular}{|c|c|c|c|c|c|}
\hline Metric & FSM & ISM & NIC & NNC & Others \\
\hline Count & $\mathrm{x}$ & $\mathrm{x}$ & $\mathrm{x}$ & $\mathrm{x}$ & $\mathrm{x}$ \\
\hline Reference Match (RM) & $x$ & & & & \\
\hline _3'_polyA_supported & $x$ & $x$ & $x$ & $x$ & \\
\hline _5'_CAGE_supported & $x$ & $x$ & $x$ & $x$ & \\
\hline _3'_reference_supported & $x$ & $x$ & $x$ & $\mathrm{x}$ & \\
\hline _5'_reference_supported & $x$ & $x$ & $x$ & $x$ & \\
\hline Supported Reference Transcript Model (SRTM) & $x$ & $x$ & & & \\
\hline Supported Novel Transcript Model (SNTM) & & & $\mathrm{x}$ & $x$ & \\
\hline Distance (nts) to TSS/TTS of matched transcript & $\mathrm{x}$ & $\mathrm{x}$ & & & \\
\hline Redundancy & $\mathrm{x}$ & $\mathrm{x}$ & & & \\
\hline$\%$ Long Read Coverage (\%LRC) & $\mathrm{x}$ & & & & \\
\hline Longest Junction Chain & & $\mathrm{x}$ & $\mathrm{x}$ & $\mathrm{x}$ & \\
\hline Intron retention level & & $\mathrm{x}$ & $\mathrm{x}$ & & \\
\hline Illumina Splice Junction Support & $x$ & $x$ & $\mathrm{x}$ & $x$ & $x$ \\
\hline Full Illumina Splice Junction Support & $\mathrm{x}$ & $\mathrm{x}$ & $\mathrm{x}$ & $\mathrm{x}$ & $\mathrm{x}$ \\
\hline$\%$ Novel Junctions & & & $\mathrm{x}$ & $\mathrm{x}$ & \\
\hline
\end{tabular}




\begin{tabular}{|l|c|c|c|c|c|}
\hline \% Non-canonical junctions & $X$ & $X$ & $X$ & $X$ & $X$ \\
\hline \% Transcripts with non-canonical junctions & $X$ & $X$ & $X$ & $X$ & $X$ \\
\hline Intra-priming & $X$ & $X$ & $X$ & $X$ & $X$ \\
\hline RT-switching & $X$ & $X$ & $X$ & $X$ & $X$ \\
\hline Number of exons & $X$ & $X$ & $X$ & $X$ & $X$ \\
\hline
\end{tabular}

662

663 High-confidence transcripts derived from LRGASP data (Positives $\mathrm{P}$ are the set of all high664 confidence transcripts)

665 Finally, a set of manually curated transcript models will be used to estimate sensitivity on real 666 data. Metrics that will be applied in this transcript set are: TP, PTP, FN, Sensitivity, Positive 667 Detection Rate, Redundancy and \%LRC.

Challenge 2 Evaluation: Transcript isoform quantification

671 We will evaluate transcript isoform quantification performance with both simulated and real 672 sequencing data, which includes SIRV-Set 4. While the ground truth is known for the simulated 673 data and SIRV-Set4, we will experimentally quantify the abundances of transcript isoforms from 674 select loci (genes) within the LRGASP samples. Specifically, we will interrogate the presence of 675 specific transcript isoforms using qPCR measurements of isoform-specific regions, and will 676 obtain such data using an aliquot of the exact same RNA which was used to generate the 677 LRGASP datasets (human and mouse).

Evaluation metrics

680 We evaluate the quantification performance for different data scenarios (Figure 3):

681 1) Single sample data when the ground truth is available

682 2) Multiple replicates under two different conditions when the ground truth is available

683 3) Multiple replicates when ground truth is not available 
685 The participants of the Challenge 2 can run these evaluations via submitting their quantification 686 results at the website https://lrrna-seq-quantification.org/ that generates an interactive report in 687 the html and PDF formats (See Data and code availability).

688

689 Single sample data (ground truth is available)

690 We can evaluate how close the estimations and the ground truth values are by four metrics as 691 follows.

692 Denote $\hat{\Theta}=\left(\hat{\theta}_{1}, \cdots, \hat{\theta}_{I}\right)^{T}$ and $\Theta=\left(\theta_{1}, \cdots, \theta_{I}\right)^{T}$ as the estimation and ground truth of the 693 abundance of $I$ transcript isoforms in a sample, respectively. Then, four metrics can be 694 calculated by the following formulas.

695 -Spearman Correlation Coefficient (SCC)

697 SCC evaluates the monotonic relationship between the estimation and the ground truth, which 698 is based on the rank for transcript isoform abundance (Supplementary Fig. S1). It is calculated 699 by

$$
S C C_{\Theta, \hat{\Theta}}=\frac{\operatorname{cov}\left(r g_{\Theta}, r g_{\hat{\Theta}}\right)}{s_{r g_{\Theta}} \cdot s_{r g_{\hat{\Theta}}}}
$$

701 where $r g_{\Theta}$ and $r g_{\hat{\Theta}}$ are the ranks of $\Theta$ and $\hat{\Theta}$, respectively, and $\operatorname{cov}\left(r g_{\Theta}, r g_{\hat{\Theta}}\right)$ is the covariance of the corresponding ranks, $s_{r g_{\Theta}}$ and ${ }^{r} g_{\hat{\Theta}}$ are the sample standard deviations of $r g_{\Theta}$ and $r g_{\hat{\Theta}}$,

703 respectively.

-Abundance Recovery Rate $(A R R)$ $A R R$ is the percentage of the estimation over the ground truth, which is calculated by An accurate abundance estimation should have an $A R R$ value close to $100 \%$.

$710 M R D$ is the median of the relative difference of abundance estimates among all transcript 711 isoforms within a sample, which is calculated by

$$
M R D=\operatorname{median}\left\{\frac{\left|\theta_{i}-\hat{\theta}_{i}\right|}{\theta_{i}}, \quad(i=1,2, \cdots, I)\right\}
$$


713 A small $M R D$ value indicates the good performance of abundance estimation.

-Normalized Root Mean Square Error (NRMSE) NRMSE provides a measure of the extent to which the one-to-one relationship deviates from a

717 linear pattern. It can be calculated by

$$
N R M S E=\frac{\sqrt{\frac{1}{I} \sum_{i=1}^{I}\left(\theta_{i}-\hat{\theta}_{i}\right)^{2}}}{s_{\Theta}}
$$

where $s_{\Theta}$ is the sample standard deviation of $\Theta$.

A good performance of abundance estimation should have a small value of NRMSE.

In the case of LRGASP, the above metrics can be calculated with simulated data and SIRVs.

\section{Multiple replicates under two different conditions (ground truth is available)}

725

Denote $\widehat{\theta}_{i j k}$ and $\theta_{i j k}$ as the estimation and ground truth of transcript isoform $i(i=1,2, \cdots, I)$ in

We assess the quantification performance by ROC (receiver operating characteristic) analysis

731 Fold Change $(A L F C)$ of transcript isoform $i$ as:

$$
A L F C_{i}=\log \left(\frac{\frac{1}{K_{2}} \sum_{k_{2}=1}^{K_{2}}\left(\theta_{i 2 k_{2}}+1\right)}{\frac{1}{K_{1}} \sum_{k_{1}=1}^{K_{1}}\left(\theta_{i 2 k_{1}}+1\right)}\right) .
$$

733 Next, based on the ground truth values and a given threshold (e.g., 1 as below), we can define 734 whether a transcript isoform is truly differentially expressed or not:

Positives (truly differentially expressed)

$$
T=\left\{i|| A L F C_{i} \mid \geq 1\right\}
$$

740 negatives" with the same threshold. Therefore, we can identify "true positives", "true negatives",

741 "false positives" and "false negatives" to calculate the ROC-based statistics, including precision,

742 recall, accuracy, F1-score, AUC and pAUC, and also plot ROC (Supplementary Fig. S2). 
744 The above metrics will be used for SIRVs and a subset of isoforms whose abundances were

745 experimentally determined. In the case of SIRV sequencing, we would not expect fold change

746 differences in different conditions, as the SIRVs were spiked in at relatively the same

747 concentration in all samples.

748

$749 \quad$ Multiple replicates under different conditions (without the ground truth)

750 For multiple replicates under different conditions without the ground truth, we can still evaluate a

751 quantification method by the "goodness" of its statistical properties, including reproducibility,

752 consistency and resolution entropy that is also calculated for single sample data

753 (Supplementary Figs. S3-S5)

754

755

-Reproducibility

756

The reproducibility statistic characterizes the average standard deviation of abundance estimates among different replicates (Supplementary Fig. S3), which is calculated by

Here, $s_{i j}$ is the sample standard deviation of $\log \left(\widehat{\theta}_{i j k}+1\right)(k=1,2, \ldots, K)$, which is 760 calculated by

$$
R M=\sqrt{\frac{1}{I J} \sum_{i=1}^{I} \sum_{j=1}^{J} s_{i j}^{2}}
$$

762 where

$$
s_{i j}=\sqrt{\frac{1}{K} \sum_{k=1}^{K}\left(\log \left(\widehat{\theta}_{i j k}+1\right)-u_{i j}\right)^{2}},
$$

With a small value of this metric, the method has high reproducibility. We can also plot $s_{i j}$

765 versus average abundance $u_{i j}$ to examine how standard deviation changes with respect to the 766 abundance and the area under the curve is calculated as a secondary statistic.

-Consistency 
A good quantification method tends to have the consistency of characterizing abundance patterns in different replicates. Here, we propose a consistency measure $C(\alpha)$ to examine the similarity of abundance profiles between mutual pairs of replicates (Supplementary Fig. S4),

772 which is defined as:

$C(\alpha)=\frac{1}{I J \cdot C_{K}^{2}} \sum_{i=1}^{I} \sum_{j=1}^{J} \sum_{1 \leq k_{1}<k_{2} \leq K}^{I} P\left(\left\{\log \left(\widehat{\theta}_{i j k_{1}}+1\right)<\alpha, \log \left(\widehat{\theta}_{i j k_{2}}+1\right)<\alpha\right\}\right.$ or $\left.\left\{\log \left(\widehat{\theta}_{i j k_{1}}+1\right) \geq \alpha, \log \left(\widehat{\theta}_{i j k_{2}}+1\right) \geq \alpha\right\}\right)$,

where $\alpha$ is a customized threshold defining whether a transcript is expressed or not.

-Resolution Entropy (RE)

A good quantification method should have a high resolution of abundance values. For a given sample, a Resolution Entropy ( $R E)$ statistic characterizes the resolution of abundance estimation (Supplementary Fig. S5):

Here, the abundance estimates are binned into $M$ groups, where $n_{m}$ represents the number of transcript isoforms with the abundance estimate $\widehat{\Theta} \in[m \cdot \alpha,(m+1) \cdot \alpha)$, and $\alpha=\max (\widehat{\Theta}) / M$. RE $=0$ if all transcript isoforms have the same estimated abundance values, while it obtains a large value when the estimates are uniformly distributed among $M$ groups.

\section{Evaluation with respect to multiple transcript features}

788 Quantification performance could be influenced by different transcript features, such as exon-

789 isoform structure and the true abundance level. Thus, we also evaluate the quantification

790 performance for different sets of genes/transcripts grouped by transcript features, including

791 number of isoforms, number of exons, ground truth abundance values and a customized

792 statistic K-value representing the complexity of exon-isoform structures.

- K-value

795 Most methods for transcript isoform quantification assign sequencing coverage to isoforms;

796 therefore, the exon-isoform structure of a gene is a key factor influencing quantification

797 accuracy. Here, we use a statistic K-value (manuscript in preparation, Supplementary Fig. S6)

798 to measure the complexity of exon-isoform structures for each gene. Suppose a gene of interest 
799

has $I$ transcript isoforms and $E$ exons, and define $A=\left(a_{i e}\right),(i=1,2, \cdots, I ; e=1,2, \cdots, E)$ as the exon-isoform binary matrix, where

$$
a_{i e}=\left\{\begin{array}{c}
1, \text { if the isoform } i \text { includes the exon } e \\
0, \text { otherwise }
\end{array}\right.
$$

$\mathrm{K}$-value is the condition number of the exon-isoform binary matrix $A$, which is calculated by

$$
\mathrm{K} \text {-value }=\frac{\sigma_{\max }(A)}{\sigma_{\min }(A)}
$$

where $\sigma_{\max }(A)$ and $\sigma_{\min }(A)$ are the maximum and minimum singular values of the matrix $A$, respectively.

With genes binned by the complexity of their transcripts, we are also able to evaluate how often the rank of isoforms from highest to lowest abundance agree between different tools, regardless of a ground truth. In particular, we can evaluate how often the most abundant isoform (major isoform) has the same transcript structure as other methods and how this compares to the ground truth, if known. We would expect more variability in what is considered the major isoform of a gene that is correlated with an increased K-value.

\section{Challenge 3 Evaluation: De novo transcript isoform detection without a high-quality genome}

Challenge 3 will evaluate the applicability of IrRNA-seq for de novo delineation of transcriptomes in non-model organisms. The evaluation will assess the capacity of technologies and analysis pipelines for both defining accurate transcript models and for correctly identifying the complexity of expressed transcripts at genomic loci, when genome information is limited. We will evaluate two different scenarios: a) availability of a genome sequence but no gene annotation is available, and b) no genome assembly is available at all.

The challenge includes three types of datasets. The mouse ES transcriptome data (Table 1) will be used to request the reconstruction of mouse transcripts without making use of the available genome or transcriptome resources for this species. Models will be compared to the true set of annotations with the same set of parameters as in Challenge 1 . While this dataset allows for a quantitative evaluation of transcript predictions in Challenge 3 , it might deliver unrealistic results if analysis pipelines were somehow biased by information derived from prior knowledge of the mouse genome. To avoid this problem, a second dataset is used that corresponds to the whole 
830 blood transcriptome of the Floridian manatee (Trichechus matatus). An lllumina draft genome of 831 this organism exists (https://www.ncbi.nlm.nih.gov/assembly/GCF 000243295.1/) and the

832 LRGASP consortium has generated a long-read genome assembly to support transcript

833 predictions for this species. Additionally, Illumina data has been generated for this challenge

834 and an existing set of 454 transcriptome data will be used. Again, we will evaluate pipelines that

835 obtain transcript models without genome annotation but with these draft genome sequences,

836 and without genome assembly data at all. Since no curated gene models exist for the manatee,

837 Challenge 1 metrics cannot be applied. Instead, the evaluation of this dataset will involve

838 comparative assessment of the reconstructed transcriptomes and experimental validation. For 839 comparative assessment the following parameters will be calculated.

a. Total number of transcripts

b. Mapping rate of transcripts to the draft genomes (for pipelines not using genome data)

c. Length of the transcript models

d. \% of transcripts with predicted coding potential

e. Does the pipeline provide gene/loci predictions? If yes, number of transcripts/loci

f. BUSCO completeness

We expect that good-performing pipelines will obtain longer transcripts, well supported by Illumina data, with high mapping rate to the draft genomes, most of them coding, and with higher BUSCO completeness and Blast2GO annotation potential.

Finally, the manatee long reads data also contain spiked-in SIRVs, which will be used to compute performance metrics for Challenge 3 analysis settings, using the same type of metrics as described for Challenge 1.

We will compare metric statistics across analysis pipelines and sequencing platforms. A number

859 of genes will be selected for PCR-based experimental validation (see below), including

860 transcripts of cytokine genes, which have been studied by LRGASP consortium members in

861 detail ${ }^{32}$. Ferrante et al. ${ }^{32}$ designed and validated primers to measure cytokine transcript levels in

862 Florida manatees from blood samples, specifically for interleukin (IL)-2, -6, -10, interferon- 
gamma (INF-gamma) and Tumor necrosis-alpha (TNF-alpha), and these methods will be

864 adopted for development of isoform-specific assays.

\section{Experimental validation of transcript models and expression estimates}

867 Independent experimental validation will be performed to assess the accuracy of novel features 868 and transcript isoforms characterized from the IrRNA-seq data from all challenges. In the 869 evaluation of full-length transcripts, several local and long-range elements must be considered. 870 Local elements include the 5' end of the transcript, splice site, junctions, novel exons, retained 871 introns, and polyA sites. Long-range elements include chained series of junctions. We will 872 employ a suite of several assays in order to validate both the local and long-range elements.

873 Challenge 1 Evaluation: Transcript isoform detection

874 The goal of this challenge is to assess the comprehensive and reliable detection of all 875 transcripts in biological samples. Similar to past studies that have employed IrRNA-seq 876 approaches towards characterizing the transcriptome, we expect that participants for this 877 challenge will produce a large number of novel isoforms. Therefore, the approaches to assess 878 the accuracy of transcript isoforms that were previously described (e.g., SIRV standards, 879 GENCODE manual annotation) will be complemented with experimental validation.

880 We will employ several high-throughput sequencing-based assays to validate local elements, 881 such as novel 5' ends, splice junctions, and polyA sites, on a "global" scale. Note that these 882 experimental assays have or will be carried out using the same aliquot of total RNA as was 883 used to generate the LRGASP datasets, minimizing differences in detected features due to 884 biological or inter-laboratory variability. To validate novel 5' ends, we will use a recently 885 generated a deep coverage CAGE data on the WTC-11 line. To validate novel splice junctions, 886 we will also use lllumina RNA-seq to validate novel junctions and, wherever possible, exons or 887 series of connected exons. To validate novel polyadenylation sites, we will collect polyA-seq 888 data using the Quant-Seq method from Lexogen, which can map polyA sites de novo.

889 Additionally, in select cases, novel 5' ends will be further corroborated through chromatin-based 890 functional information derived from ENCODE data, such as the presence of Polll or histone 891 marks that are indicative of active promoters.

892 Longer-range features within a transcript, such as chains of junctions, are difficult and 893 sometimes impossible to detect through short-read sequencing approaches or traditional qPCR; 
894 therefore, we will employ targeted amplicon sequencing followed by ONT, PacBio, and Sanger 895 sequencing.

896 We plan to select 96 targets from human WTC-11 cells and 96 targets from the mouse 897 129/Casteneus cells. Each target will comprise a sequence region 300 to 1500 bp long. Two 898 replicates each from the WTC-11 and 129/Casteneus sample will be apportioned for a reverse899 transcriptase reaction followed by target amplification using isoform-specific primers. We will 900 conduct the assay in plate format to allow for high-throughput processing. All products following 901 RT-PCR will be pooled and subjected to long-read sequencing for validation. A subset of these 902 samples will be selected for Sanger sequencing. Table 6 shows the breakdown of targets we 903 will select.

904

\begin{tabular}{|l|l|l|}
\hline Category & WTC-11 (Human) & $\begin{array}{l}\text { 129/Casteneus } \\
\text { (Mouse) }\end{array}$ \\
\hline Positive control & 12 & 12 \\
\hline Negative control & 12 & 12 \\
\hline Novel - detected in all platforms & 12 & 12 \\
\hline $\begin{array}{l}\text { ONT-specific } \\
\text { PacBio-specific }\end{array}$ & 12 & 12 \\
\hline $\begin{array}{l}\text { Miscellaneous category (e.g., bioinformatic } \\
\text { pipeline-specific, intron retention, template } \\
\text { switch artifact prediction, non-canonical } \\
\text { splicing) }\end{array}$ & 24 & 12 \\
\hline
\end{tabular}

905 Table 6: Plan for targeted amplicon sequencing to validate novel junction chains in the 906 LRGASP submissions.

907

908 Positive controls will be selected as subsegments of isoforms which are found in GENCODE 909 human v39 and mouse vM28, all long-read datasets across the ONT and PacBio platforms, and 910 a majority (>50\%) of the computational pipelines. Negative controls will also be selected, which 
911 would involve isoforms that are detected in other human and mouse cell types (e.g., pancreas

912 cells), but for which there is no evidence of expression across any of the long-read datasets in

913 LRGASP.

914 An open question in the field is the accuracy of novel isoforms that are frequently detected on

915 long-read platforms, and so we will devote substantial effort towards validation of novel

916 isoforms. At least 12 targets will involve junction chains that are novel (not in GENCODE) but

917 found across all IrRNA-seq library types. We also reserve resources to validate platform-specific

918 isoforms, in case they should arise. And, lastly, we reserve at least 24 targets for miscellaneous

919 categories, such as if there is the appearance of certain isoforms in specific computational

920 pipelines.

921 For novel target selection, preference will be given to select targets that correspond to the pre-

922 selected 50 loci that will be manually annotated by GENCODE, and there will be close

923 coordination between the working groups.

924 In addition to the validation using a PCR-based approach (Table 6), high-throughput validation

925 of full-length transcripts will be obtained by application of the NRCeq strategy ${ }^{25}$ on WTC-11

926 cells, which does not rely on PCR. NRCeq employs a chemical labeling strategy to add a

927 signature oligonucleotide exclusively to the 5' caps of mRNAs, thus, full-length mRNA

928 sequences from the 5' cap to the polyA sequence may be distinguished from incomplete

929 sequence fragments. We will compare NRCeq data generated WTC-11 against models

930 submitted by participants.

931 Challenge 2 Evaluation: Transcript isoform quantification

932 Challenge 2 involves the prediction of fold change in abundance at the gene and transcript

933 isoform-level. For this purpose, the H1:H1-DE cell line mix will be compared to WTC11 cell line.

$934 \mathrm{H} 1$ and WTC-11, both being stem cell lines, are expected to have similar expression patterns,

935 but the $\mathrm{H} 1: \mathrm{H} 1-\mathrm{DE}$ mix would have gene and isoform expression more related to the definitive

936 endoderm phenotype. To experimentally validate abundance changes, we will employ qPCR

937 among isoforms of a gene which under altered expression as well as sequencing data on

938 sample components before mixing.

939

940 qPCR of 10-20 transcript models will be performed. Due to the difficulty of properly resolving

941 and apportioning signals for short junctions or exons to the full-length transcript isoforms they

942 arose from, we will choose isoforms with low and high K-values, representing various levels of 
943 identifiability. In some cases, we will increase the length of qPCR targets up to the 500-600 bp

944 ranges so as to increase the resolution and specificity of isoform measurements. Internal

945 standards will be spiked in for highest accuracy and precision of isoform abundance

946 estimates.Targeted amplicon sequencing with long-read platforms will also be performed on

947 these transcript models to determine fold-change differences.

948 Due to the challenges of isoform-level quantification and the lack of a gold standard, we devised 949 a mixture sample, in which an undisclosed ratio of two samples is mixed before sequencing. For 950 validation, we sequenced $\mathrm{H} 1$ and $\mathrm{H} 1-\mathrm{DE}$ samples individually to establish the isoforms present 951 in only one or the other sample before mixing. In essence, the pre-mixed sample represents the 952 "ground truth" of isoform expression before the mix. After the close of LRGASP submissions, 953 the $\mathrm{H} 1$ and $\mathrm{H} 1-\mathrm{DE}$ long-read data will be released. Participants of Challenge 2, will need to 954 provide transcript quantification from these additional datasets. Libraries and computational 955 pipelines can then be evaluated based on how well the transcript quantification in the H1:H1-DE 956 mix sample represents the expected ratios determined from quantification from the individual 957 cell lines.

958 Challenge 3 Evaluation: De-novo transcript isoform detection without a high-quality genome

959 Similarly to Challenge 1, the primary goal of experimental validation in this challenge is to 960 confirm the identity of de novo assembled isoforms, of which many will be novel.

961 A number of loci from well-studied immune-related genes will be selected for experimental PCR 962 validation as in the mouse/human data.

963 To validate isoforms containing novel junction chains, we will employ a similar amplicon 964 sequencing strategy as described in Challenge 1, in which up to 96 primer pairs will be used to 965 amplify isoform-specific regions for subsequent detection on a sequencing platform.

966 In addition, there exists 454 sequencing data from these same samples which can also be 967 leveraged for orthogonal validation.

\section{Challenge submissions and timeline}

969 Participants will submit challenge predictions on Synapse

970 (https://www.synapse.org/\#!Synapse:syn25007472).

972 The following is an overview of the data used for each challenge and the result files that will be 973 submitted (Supplementary Figure S7). 
- Challenge 1: transcript isoform detection with a high-quality genome (iso_detect_ref)

- WTC11 (human iPSC cell line)

- H1_mix (human H1 ES cell line mixed with human Definitive Endoderm derived from $\mathrm{H} 1$ )

- ES (mouse ES cell line)

- human_simulation - simulated human reads (Illumina, ONT, and PacBio cDNA)

- mouse_simulation - simulated mouse reads (Illumina and PacBio cDNA, ONT dRNA)

- Result files:

- models.gtf.gz

- read_model_map.tsv.gz

- Challenge 2: transcript isoform quantification (iso_quant)

- WTC11 (human iPSC cell line)

- H1_mix (human H1 ES cell line mixed with human Definitive Endoderm derived from $\mathrm{H}$ 1)

- human_simulation - simulated human reads (Illumina, ONT, and PacBio cDNA)

- mouse_simulation - simulated mouse reads (Illumina and PacBio cDNA, ONT dRNA)

- Result files:

- expression.tsv.gz

1002

1003

1004

1005

- Challenge 3: de novo transcript isoform detection (iso_detect_de_novo)

- Samples

- Manatee (manatee whole blood)

- ES (mouse ES cell line)

○ Result files:

- rna.fasta.gz

- read_model_map.tsv.gz 
A submission to a challenge is an entry, consisting of one or more experiments. Each entry must meet the following requirements:

1008

Requirements for Challenge 1 and 2

At least one experiment must be supplied for each sample available for a given challenge. Human and mouse samples will have biological replicates that should be used for the entry.

A major goal of LRGASP is to assess the capabilities of long-read sequencing for transcriptome analysis and also how much improvement there is over short-read methods. Additionally, longread computational pipelines vary in their use of only long-read data or if they incorporate additional data for transcript analysis. To facilitate comparisons between long-read and shortread methods and variation in tool parameters, we break down submissions into different categories:

- long-only - Use only LGRASP-provided long-read RNA-Seq data from a single sample, library preparation method and sequencing platform.

- short-only - Use only LGRASP-provided short-read Illumina RNA-Seq data from a single sample. This is to compare with long-read approaches

- long and short - Use only LGRASP-provided long-read and short-read RNA-Seq data from a single long-read library preparation method and the Illumina platform. Additional accessioned data in public genomics data repositories can also be used.

- kitchen sink - Any combination of at least one LRGASP data set as well as any other accessioned data in public genomics data repositories. For example, multiple library methods can be combined (e.g. PacBio cDNA + PacBio CapTrap, ONT cDNA + ONT CapTrap+ ONT R2C2+ ONT dRNA, all data, etc.).

All replicates must be used in each experiment. Challenge 2 must report replicates separately in 1037

In all the above categories, the genome and transcriptome references specified by LRGASP should be used. For the long and short and kitchen sink category, additional transcriptome references can be used. the expression matrix. Each team can only submit one entry per category.

For Challenge 1, the submitted GTF file should only contain transcripts that have been assigned a read. For Challenge 2, submitters have the option of quantifying against the reference 
1040 transcriptome or a transcriptome derived from the data (i.e., results from Challenge 1). The GTF used for quantification is included as part of the Challenge 2 submission.

The type of platform and libraries preparation method used in a given experiment, except for kitchen sink experiments, is limited to data from a single library preparation method plus sequencing technology (long-only). LRGASP Illumina short-read data of the same sample may optionally be used in an experiment with the LRGASP long-read data (long and short)

- Illumina cDNA - short-only

Requirements for Challenge 3

At least one experiment must be supplied for each sample available for the challenge. Mouse samples will have biological replicates that should be used for the entry. For similar reasons as described above, the data used for a given experiment must fit in one of the following categories:

- long-only - Use only LGRASP-provided long-read RNA-Seq data from a single sample, library preparation method and sequencing platform. No genome reference can be used.

- short-only - Use only LGRASP-provided short-read Illumina RNA-Seq data from a single sample. This is to compare with long-read approaches. No genome reference can be used.

- long and short - Use only LGRASP-provided long-read and short-read RNA-Seq data from a single long-read library preparation method and the Illumina platform. No genome reference can be used.

- long and genome - Use only LGRASP-provided long-read RNA-Seq data from a single long-read library preparation method. A genome reference sequence can be used.

- kitchen sink - Any combination of at least one LRGASP data set as well as any other accessioned data in public genomics data repositories. For example, multiple library methods can be combined (e.g. PacBio cDNA + PacBio CapTrap, ONT cDNA + ONT CapTrap+ ONT R2C2+ ONT dRNA, all data, etc.). 
1075 In all the above categories, except for kitchen sink a transcriptome reference cannot be used.

1076 The submitted FASTA file should only contain transcripts that have been assigned a read.

1077 Each team can only submit one entry per category.

1078

LRGASP biological data is currently available at the ENCODE DCC simulated data is available from Synapse (https://www.synapse.org/\#!Synapse:syn25683370). The competition launched on May 1, 2021 and challenge submissions are expected to close on October 1, 2021.

\section{LRGASP Data QC}

Initial quality control (QC) metrics were determined for the LRGASP data (Figure 4). Reads (ONT cDNA, dRNA, CapTrap) or consensus reads (PacBio cDNA and CapTrap and ONT $\mathrm{R} 2 \mathrm{C} 2$ ) were aligned to the human or mouse genome as appropriate using minimap2 with the following parameters: -ax splice --secondary=no -G 400k. For each data type, the reads and their resulting alignments in sam format were parsed for the following parameters:

1) Number of aligned reads

2) Number of aligned reads with adapters on both ends

For ONT dRNA this is not applicable as this workflow does not attach an adapter to the 5' end of molecules. For ONT cDNA and CapTrap this percentage was determined by pyChopper. For all other data types, all provided reads are assumed to have adapters on both ends as the pre-processing pipelines (lima and $\mathrm{C} 3 \mathrm{PO}$ ) discard reads otherwise.

3) median read length measured by the number of aligned bases (matches or mismatches)

4) median accuracy measured by matches/(matches+mismatches+indels)), 

sequence files determined by quantifying gene expression for each replicate and calculating the pearson $r$ value based on those expression values.

1114 Table 7: Summary statistics for LRGASP data. For each sample, replicates were combined 1115 when reporting statistics.

1116

\begin{tabular}{|c|c|c|c|c|c|c|}
\hline Sample & ES & & & & & \\
\hline Method & dRNA & cDNA & $\mathrm{R} 2 \mathrm{C} 2$ & CapTrap & CapTrap & cDNA \\
\hline Tech & ONT & ONT & ONT & ONT & PacBio & PacBio \\
\hline Platform & MinlON & MinION & MinION & MinION & Sequelll & Sequelll \\
\hline \# of Flowcells/SMRT cells & 3 & 3 & 6 & 3 & 3 & 9 \\
\hline \# of raw reads & $4,325,200$ & $59,746,818$ & $7,862,883^{1}$ & $56,684,765$ & $9,689,619$ & $23,487,808$ \\
\hline \# of supplied reads & $3,975,725$ & $57,055,583$ & $5,930,487$ & $50,697,997$ & $5,090,848$ & $8,733,814$ \\
\hline \# of aligned reads & $3,836,020$ & $44,873,564$ & $5,914,779$ & $49,741,194$ & $5,028,403$ & $8,199,908$ \\
\hline $\begin{array}{l}\text { \# of aligned reads with } \\
\text { adapters }\end{array}$ & $\mathrm{N} / \mathrm{A}$ & $40,190,805$ & $5,914,779$ & $32,206,495$ & $5,028,403$ & $8,199,908$ \\
\hline Median Read length & 830 & 519 & 1,755 & 591 & 903 & 2,090 \\
\hline Median Identity (Q score) & 9.8 & 12.7 & 18.6 & 12.3 & 21.3 & 20.9 \\
\hline$\%$ Directionality & 99.54 & 98.59 & 99.74 & 94.66 & 99.88 & 99.55 \\
\hline$\%$ of spike-in reads & 0.71 & 1.02 & 2.03 & 2.41 & 1.77 & 1.85 \\
\hline Pearson r2 (gene level) & 0.99 & 0.99 & 0.98 & 0.99 & 0.98 & 0.97 \\
\hline
\end{tabular}




\begin{tabular}{|l|l|l|l|l|l|r|}
\hline Sample & WTC11 & & & & & \\
\hline Method & dRNA & CDNA & R2C2 & CapTrap & CapTrap & cDNA \\
\hline Tech & ONT & ONT & ONT & ONT & PacBio & PacBio \\
\hline Platform & MinION & MinION & MinION & MinION & Sequell & SequellI \\
\hline \# of Flowcells/SMRT cells & 3 & 3 & 6 & 3 & 3 & 9 \\
\hline \# of raw reads & $3,229,571$ & $53,463,774$ & $6,994,7891$ & $56,730,485$ & $13,463,712$ & $28,567,150$ \\
\hline \# of supplied reads & $\mathbf{2 , 9 8 8 , 4 3 0}$ & $\mathbf{5 1 , 1 9 4 , 5 3 5}$ & $\mathbf{5 , 2 7 5 , 7 3 7}$ & $\mathbf{5 0 , 9 0 2 , 3 0 3}$ & $\mathbf{6 , 3 9 9 , 6 3 2}$ & $\mathbf{7 , 4 2 4 , 9 2 3}$ \\
\hline \# of aligned reads & $2,931,482$ & $43,085,527$ & $5,271,334$ & $49,930,350$ & $6,304,610$ & $7,373,147$ \\
\hline \# of aligned reads with adapters & N/A & $37,275,068$ & $5,271,334$ & $31,348,191$ & $6,304,610$ & $7,373,147$ \\
\hline Median Read length & 854 & 610 & 1,802 & 564 & 864 & 2,209 \\
\hline Median Identity (Q score) & 9.8 & 12.9 & 19.3 & 12.9 & 22.5 & 23.8 \\
\hline \% Directionality & 99.76 & 99.11 & 99.92 & 96.28 & 99.92 & 99.67 \\
\hline \% of spike-in reads & 0.6 & 1.45 & 2.27 & 2.79 & 2.26 & 2.25 \\
\hline Pearson r2 (gene level) & 0.92 & 0.96 & 0.94 & 0.99 & 0.96 & 0.90 \\
\hline $\begin{array}{l}\text { 1.R2C2 libraries for ES and WTC11 libraries were multiplexed and raw reads cannot be demultiplexed directly. Raw read numbers for these } \\
\text { libraries are therefore calculated based on the ES/WTC11 ratio of demultiplexed supplied consensus reads and total number of subreads. }\end{array}$ \\
\hline
\end{tabular}

\begin{tabular}{|c|c|c|c|c|c|c|}
\hline Sample & H1_mix & & & & & \\
\hline Method & dRNA & cDNA & $\mathrm{R} 2 \mathrm{C} 2$ & CapTrap & CapTrap & cDNA \\
\hline Tech & ONT & ONT & ONT & ONT & PacBio & PacBio \\
\hline Platform & MinION & MinION & MinION & MinION & Sequelll & Sequelll \\
\hline \# of Flowcells/SMRT cells & 3 & 3 & 6 & 3 & 3 & 6 \\
\hline \# raw reads & $4,223,164$ & $55,927,828$ & $7,093,671$ & $54,055,468$ & $10,534,880$ & $24,290,762$ \\
\hline \# of supplied reads & $3,969,603$ & $52,927,595$ & $5,231,255$ & $49,883,469$ & $5,511,853$ & $5,511,357$ \\
\hline \# of aligned reads & $3,905,742$ & $43,026,016$ & $5,229,686$ & $48,424,901$ & $5,436,170$ & $5,480,635$ \\
\hline \# of aligned reads with adapters & $\mathrm{N} / \mathrm{A}$ & $36,653,422$ & $5,229,686$ & $28,099,080$ & $5,436,170$ & $5,480,635$ \\
\hline Median Read length & 891 & 619 & 1,782 & 604 & 1,036 & 2,376 \\
\hline Median Identity (Q score) & 10.0 & 12 & 18.7 & 12.4 & 24.3 & 23.7 \\
\hline$\%$ Directionality & 99.8 & 99.19 & 99.74 & $76.15^{1}$ & 99.91 & 99.63 \\
\hline$\%$ of spike-in reads & 0.77 & 1.5 & 1.69 & 1.59 & 1.33 & 1.97 \\
\hline Pearson r2 (gene-level) & 0.99 & 0.997 & 0.98 & 0.96 & 0.98 & 0.98 \\
\hline
\end{tabular}




\begin{tabular}{|l|l|l|}
\hline Sample & Manatee & Manatee \\
\hline Method & CDNA & \multicolumn{1}{l|}{ cDNA } \\
\hline Tech & ONT & \multicolumn{1}{l|}{ PacBio } \\
\hline Platform & MinlON & \multicolumn{1}{l|}{$\begin{array}{l}\text { Sequel I + } \\
\text { Sequel II }\end{array}$} \\
\hline \# of Flowcells/SMRT cells & 3 & $1+3$ \\
\hline \# of supplied reads & $\mathbf{4 0 , 9 4 8 , 5 7 1}$ & $\mathbf{6 , 8 8 3 , 6 8 4}$ \\
\hline \# of aligned reads & $32,833,840$ & $6,877,181$ \\
\hline \# of aligned reads with adapters & $27,381,394$ & $6,877,181$ \\
\hline Median Read length & 540 & 894 \\
\hline Median Accuracy (Q score) & 12.5 & 25.2 \\
\hline \% Directionality & 97.2 & 99.76 \\
\hline \% of spike-in reads & $\mathbf{1 4 . 0 5 *}$ & $\mathbf{3 3 . 7 8 *}$ \\
\hline *spike-in percentage is higher than expected & & \\
\hline
\end{tabular}

1135

1136

\section{Data and code availability}

1138 All code and documentation associated with the LRGASP Consortium can be found through 1139 https://www.gencodegenes.org/pages/LRGASP/ and https://github.com/LRGASP. 


\section{Acknowledgments}

1146 We thank Lexogen, Oxford Nanopore Technologies (ONT), and Pacific Biosciences for helpful

1147 discussions. ONT provided partial support of flow cells and reagents. We thank Xingjie Ren and

1148 Yin Shen for providing WTC11 cells, Takayo Sasaki and Dave Gilbert for providing the F121-9

1149 hybrid mouse ES cells, and Alyssa Cousineau, Krishna Mohan Parsi, and Rene Maehr for

1150 providing human $\mathrm{H} 1$ and $\mathrm{H} 1-\mathrm{DE}$ cells. We also thank Mark Akeson and Miten Jain for providing

1151 resources and technical advice for Nanopore sequencing. We thank Julie Visser for contributing

1152 artwork that gives an overview of the LRGASP Consortium. The project is supported by the

1153 following grants: Pew Charitable Trust (A.N.B.), NIGMS R35GM138122(A.N.B.), NHGRI

1154 U41HG007234 (J.L, M.D., R.G. and S.C-S) and UM1 HG009443 (A.M. and B.W.), an

1155 institutional fund of the Department of Biomedical Informatics, The Ohio State University

1156 (K.F.A.), NHGRI R01HG008759 (K.F.A.), SPBU 73023672 (A.P). J.E.L., J.M.M. and A.F. are

1157 supported by National Human Genome Research Institute of the National Institutes of Health

1158 [U41HG007234]; the content is solely the responsibility of the authors and does not necessarily

1159 represent the official views of the National Institutes of Health; Wellcome Trust

1160 [WT108749/Z/15/Z, WT200990/Z/16/Z]; European Molecular Biology Laboratory. We

1161 acknowledge Ellie Schiller Homosassa Springs Park for providing archive Lorelei blood

1162 samples.

\section{Competing Interests}

1164 Design of the project was discussed with Oxford Nanopore Technologies (ONT), Pacific

1165 Biosciences, and Lexogen. ONT provided partial support of flow cells and reagents. S.C-S and

1166 A.N.B. have received reimbursement for travel, accommodation and conference fees to speak

1167 at events organised by ONT.

1168 
1169 1. Au, K. F. et al. Characterization of the human ESC transcriptome by hybrid sequencing.

$1170 \quad$ Proc. Natl. Acad. Sci. U. S. A. 110, E4821-30 (2013).

1171 2. Sharon, D., Tilgner, H., Grubert, F. \& Snyder, M. A single-molecule long-read survey of the 1172 human transcriptome. Nat. Biotechnol. 31, 1009-1014 (2013).

1173 3. Weirather, J. L. et al. Comprehensive comparison of Pacific Biosciences and Oxford $1174 \quad$ Nanopore Technologies and their applications to transcriptome analysis. F1000Res. 6, 100 $1175 \quad$ (2017).

1176 4. Garalde, D. R. et al. Highly parallel direct RNA sequencing on an array of nanopores. Nat. $1177 \quad$ Methods 15, 201-206 (2018).

1178 5. Byrne, A., Cole, C., Volden, R. \& Vollmers, C. Realizing the potential of full-length 1179 transcriptome sequencing. Philos. Trans. R. Soc. Lond. B Biol. Sci. 374, 20190097 (2019).

1180 6. Oikonomopoulos, S. et al. Methodologies for Transcript Profiling Using Long-Read $1181 \quad$ Technologies. Front. Genet. 11, 606 (2020).

1182 7. Rhoads, A. \& Au, K. F. PacBio Sequencing and Its Applications. Genomics Proteomics $1183 \quad$ Bioinformatics 13, 278-289 (2015).

1184 8. Hardwick, S. A., Joglekar, A., Flicek, P., Frankish, A. \& Tilgner, H. U. Getting the Entire 1185 Message: Progress in Isoform Sequencing. Front. Genet. 10, 709 (2019).

1186 9. Engström, P. G. et al. Systematic evaluation of spliced alignment programs for RNA-seq 1187 data. Nat. Methods 10, 1185-1191 (2013).

1188 10. Steijger, T. et al. Assessment of transcript reconstruction methods for RNA-seq. Nat. $1189 \quad$ Methods 10, 1177-1184 (2013).

1190 11. Reese, M. G. et al. Genome annotation assessment in Drosophila melanogaster. Genome $1191 \quad$ Res. 10, 483-501 (2000).

1192 12. Guigó, R. et al. EGASP: the human ENCODE Genome Annotation Assessment Project. 1193 Genome Biol. 7 Suppl 1, S2.1-31 (2006).

1194 13. Volden, R. et al. Improving nanopore read accuracy with the R2C2 method enables the 
sequencing of highly multiplexed full-length single-cell cDNA. Proc. Natl. Acad. Sci. U. S. A. 115, 9726-9731 (2018).

14. Carninci, P. et al. High-efficiency full-length cDNA cloning by biotinylated CAP trapper. Genomics 37, 327-336 (1996).

15. Frankish, A. et al. GENCODE 2021. Nucleic Acids Res. 49, D916-D923 (2021).

1200 16. Foote, A. D. et al. Convergent evolution of the genomes of marine mammals. Nat. Genet. 47, 272-275 (2015).

17. Picelli, S. et al. Full-length RNA-seq from single cells using Smart-seq2. Nat. Protoc. 9, 171-181 (2014).

18. Carninci, P. \& Hayashizaki, Y. High-efficiency full-length cDNA cloning. Methods Enzymol. 303, 19-44 (1999).

19. Shibata, Y. et al. Cloning full-length, cap-trapper-selected cDNAs by using the single-strand linker ligation method. Biotechniques 30, 1250-1254 (2001).

20. Tardaguila, M. et al. SQANTI: extensive characterization of long-read transcript sequences

21. Li, B. \& Dewey, C. N. RSEM: accurate transcript quantification from RNA-Seq data with or without a reference genome. BMC Bioinformatics 12, 323 (2011).

1213 22. Jo, J. et al. Midbrain-like Organoids from Human Pluripotent Stem Cells Contain Functional 1214 Dopaminergic and Neuromelanin-Producing Neurons. Cell Stem Cell 19, 248-257 (2016).

1215 23. Hafezqorani, S. et al. Trans-NanoSim characterizes and simulates nanopore RNA1216 sequencing data. Gigascience 9, (2020).

1217 24. Takahashi, H., Nishiyori-Sueki, H., Ramilowski, J. A., Itoh, M. \& Carninci, P. Low Quantity 1218 single strand CAGE (LQ-sSCAGE) maps regulatory enhancers and promoters. 1219 doi:10.1101/2020.08.04.231969.

1220 25. Mulroney, L. et al. Identification of high confidence human poly(A) RNA isoform scaffolds 
using nanopore sequencing. doi:10.1101/2020.11.18.389049.

1222 26. Schulz, L. et al. Direct long-read RNA sequencing identifies a subset of questionable exitrons likely arising from reverse transcription artifacts. Genome Biol. 22, 190 (2021).

27. Harrow, J. et al. GENCODE: the reference human genome annotation for The ENCODE Project. Genome Res. 22, 1760-1774 (2012).

28. Searle, S. M. J., Gilbert, J., lyer, V. \& Clamp, M. The otter annotation system. Genome Res. 14, 963-970 (2004).

29. Sonnhammer, E. L. \& Durbin, R. A workbench for large-scale sequence homology analysis.

1229 Comput. Appl. Biosci. 10, 301-307 (1994).

1230 30. Sonnhammer, E. L. \& Durbin, R. An expert system for processing sequence homology data. Proc. Int. Conf. Intell. Syst. Mol. Biol. 2, 363-368 (1994).

31. Sonnhammer, E. L. \& Durbin, R. A dot-matrix program with dynamic threshold control suited for genomic DNA and protein sequence analysis. Gene 167, GC1-10 (1995).

32. Ferrante, J. A., Hunter, M. E. \& Wellehan, J. F. X. DEVELOPMENT AND VALIDATION OF QUANTITATIVE PCR ASSAYS TO MEASURE CYTOKINE TRANSCRIPT LEVELS IN THE FLORIDA MANATEE ( TRICHECHUS MANATUS LATIROSTRIS). J. WildI. Dis. 54, 283294 (2018). 
a

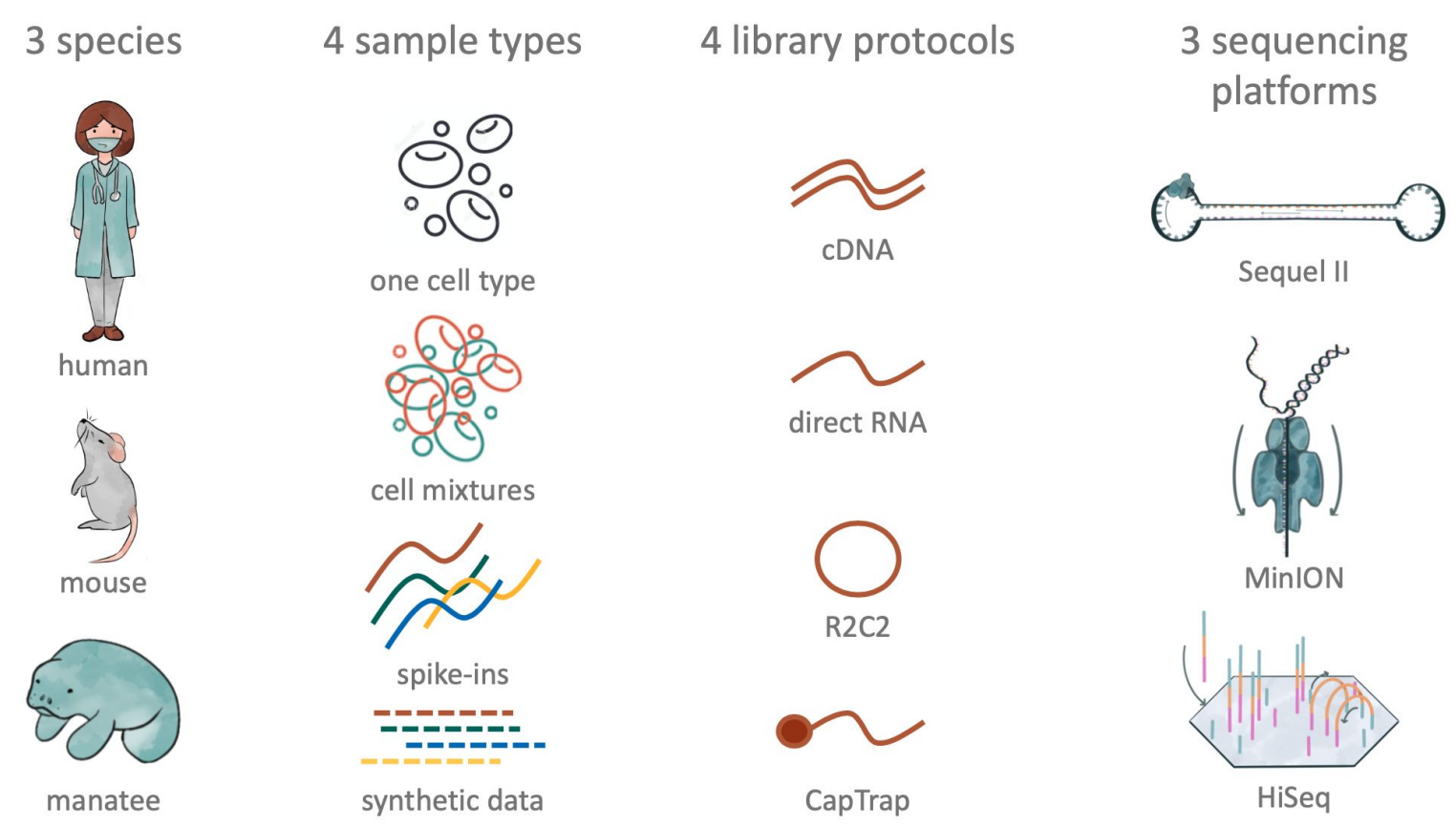

b

\begin{tabular}{|c|c|c|c|}
\hline $\begin{array}{l}\text { Sequencing data } \\
\text { provided by } \\
\text { LRGASP Organizers } \\
\text { (No embargo) }\end{array}$ & $\begin{array}{l}\text { Participants from } \\
\text { broader research } \\
\text { community }\end{array}$ & $\begin{array}{l}\text { Submission of transcript } \\
\text { assembly and } \\
\text { quantification } \\
\text { predictions }\end{array}$ & $\begin{array}{l}\text { Evaluation and } \\
\text { experimental validation } \\
\text { by LRGASP Organizers } \\
\text { with community input }\end{array}$ \\
\hline
\end{tabular}

Fig. 1: Overview of the Long-read RNA-seq Genome Annotation Assessment Project (LRGASP). a, LRGASP Consortium as a research community effort. b, Overview of LRGASP data. 
a

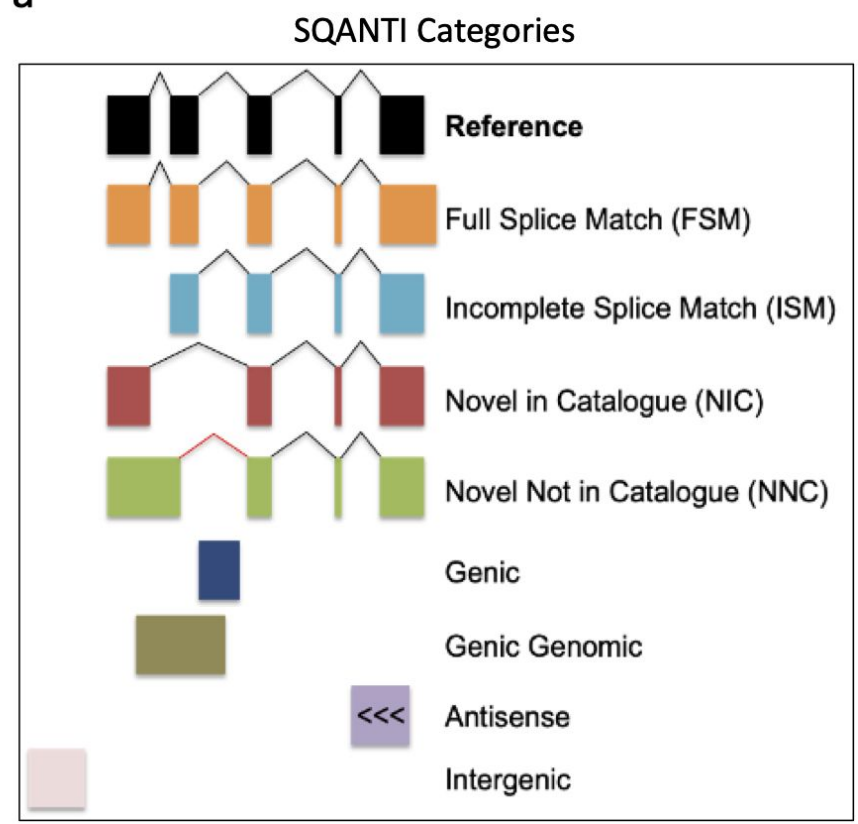

b

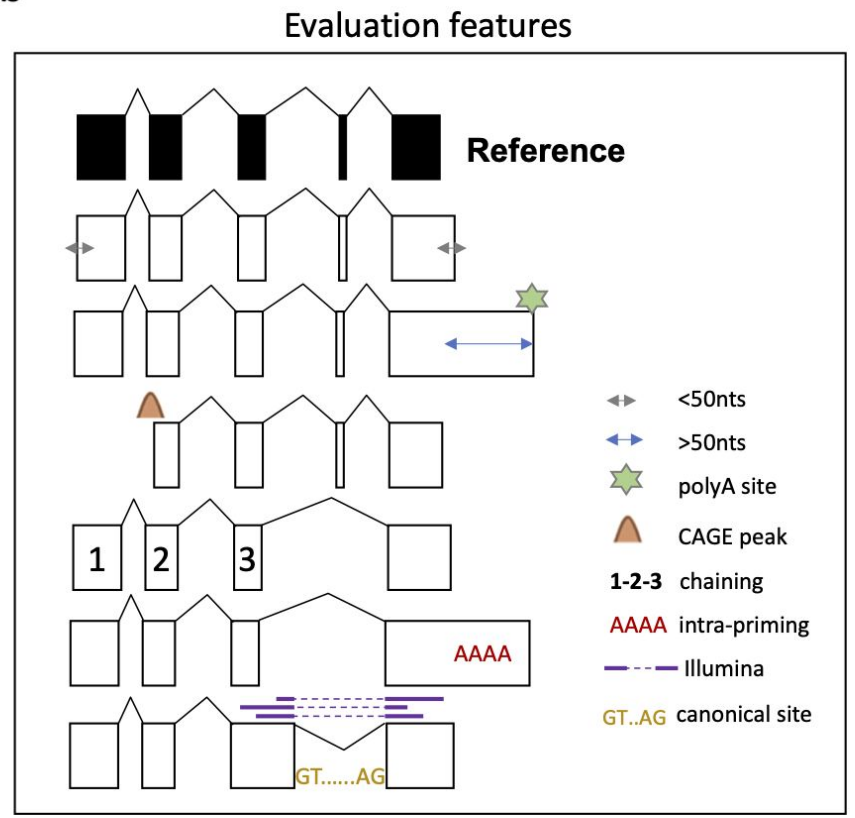

Fig. 2: SQANTI-based evaluation of transcript identification methods for Challenges 1 and 3. a, Transcripts are compared to a best matched reference transcript and categorized based on shared junctions between the reference. $\mathbf{b}$, Additional features that are considered when evaluating transcript models 


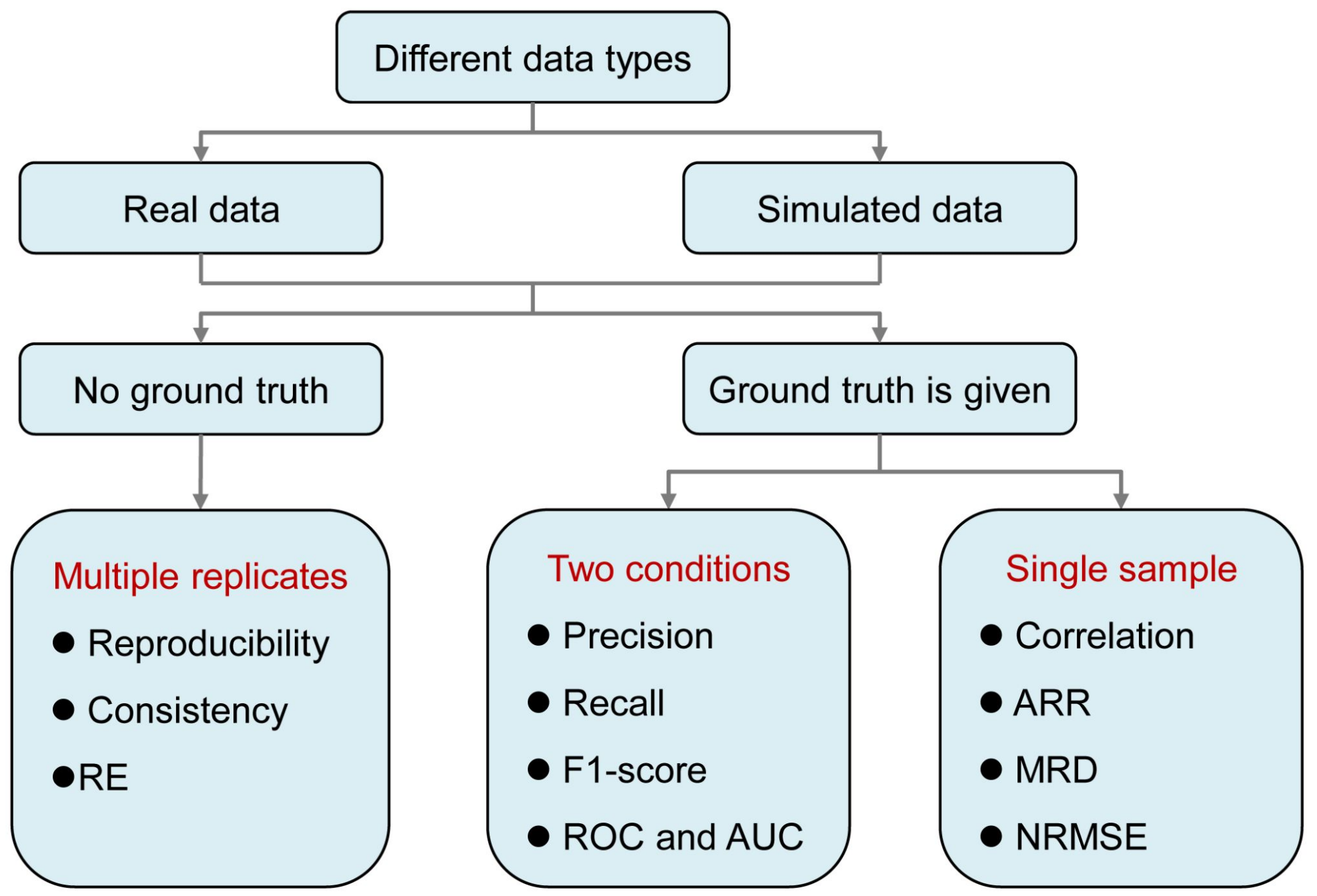

Fig. 3: Evaluation metrics of gene isoform quantification under different data types. RE - Resolution Entropy, ARR - Abundance Recovery Rate, MRD - Median Relative Difference, NRMSE - Normalized Root Mean Square Error 

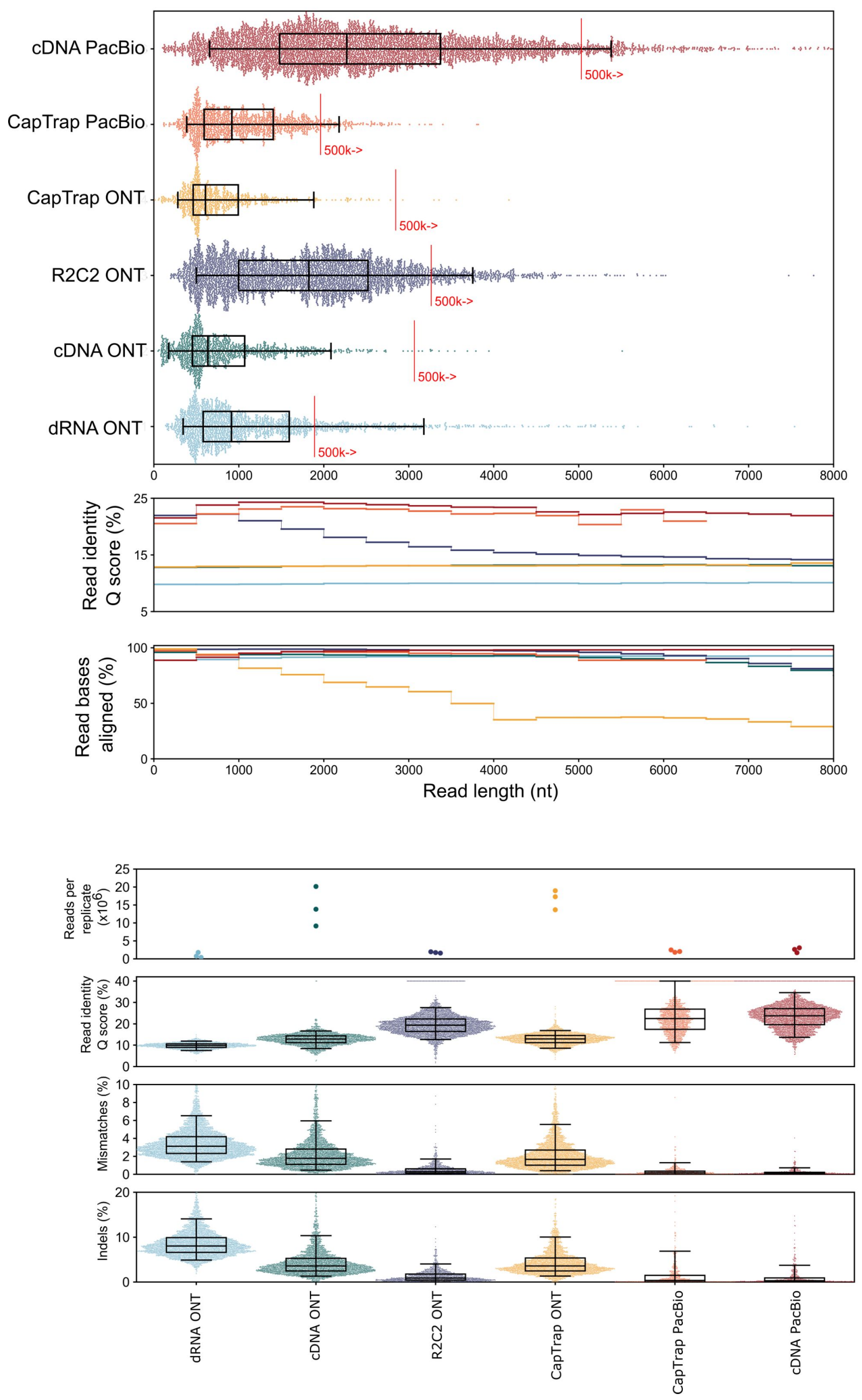

Fig. 4: Summary of LRGASP Data 


\section{Supplementary Files}

This is a list of supplementary files associated with this preprint. Click to download.

- SupplementaryTable1.txt

- RegisteredReportSupplnfo210730wFigures.pdf

- RegisteredReportSuppInfo210730wFigures.pdf 